2

3

4

\title{
COPII Sec23 proteins form isoform-specific ER exit sites with differential effects on polarized growth
}

\author{
Mingqin Chang1,2,3, Shu-Zon $\mathrm{Wu}^{1}$, Samantha E. Ryken ${ }^{1}$, Jacquelyn E. O'Sullivan ${ }^{4,5}$ \\ and Magdalena Bezanilla ${ }^{*}$
}

1 Department of Biological Sciences, Dartmouth College, Hanover, NH 03755

2 Plant Biology Graduate Program, University of Massachusetts Amherst, Amherst, MA 01002

${ }^{3}$ Current address: Department of Plant Sciences, University of California Davis, Davis, CA 95616

4 Department of Biology, University of Massachusetts Amherst, Amherst, MA 01002

${ }^{5}$ Current address: Tufts School of Medicine, Boston, MA 02111

*Corresponding author

email: magdalena.bezanilla@dartmouth.edu

The author responsible for distribution of materials integral to the findings presented in this article in accordance with the policy described in the Instructions for Authors (www.plantcell.org) is: Magdalena Bezanilla (magdalena.bezanilla@dartmouth.edu). 


\section{$31 \quad$ Abstract}

COPII, a coat of proteins that form vesicles on the ER, mediates vesicle

33 traffic from the ER to the Golgi. In contrast to metazoans that have few genes

34 encoding each COPII component, plants have expanded these gene families

35 leading to the hypothesis that plant COPII has functionally diversified. Here, we

36 analyzed the gene families encoding for the Sec23/24 heterodimer in the moss

37 Physcomitrium (Physcomitrella) patens. In P. patens, Sec23 and Sec24 gene

38 families are each comprised of seven genes. Silencing the Sec23/24 genes

39 revealed isoform specific contributions to polarized growth, with the closely

40 related $\operatorname{Sec} 23 D / E$ and $\operatorname{Sec} 24 C / D$ essential for protonemal development. Focusing

41 on the Sec23 gene family, we discovered that loss of Sec23D alters ER

42 morphology, increases ER stress, inhibits trafficking to the Golgi and to the

43 plasma membrane in tip growing protonemata. In contrast, the remaining five

$44 \operatorname{Sec} 23$ genes are dispensable for tip growth. While $\operatorname{Sec} 23 A / B / C / F / G$ do not

45 quantitatively affect ER to Golgi trafficking in protonemata, they do contribute to

46 secretion to the plasma membrane. Of the three highly expressed Sec23 isoforms

47 in protonemata, Sec23G forms ER exit sites that are larger than Sec23B and

48 Sec23D and do not overlap with Sec23D. Furthermore, ER exit sites labeled by

49 Sec23B or Sec23G form in the absence of Sec23D. These data suggest that

$50 \mathrm{Sec} 23 \mathrm{D} / \mathrm{E}$ form unique ER exit sites contributing to secretion that is essential for

51 tip growing protonemata. 


\section{Introduction}

Trafficking from the ER to the Golgi is mediated by the Coat Protein complex II (COPII). COPII facilitates the formation of transport vesicles at ER exit sites promoting the delivery of cargo, including transmembrane proteins, soluble proteins and lipids, to cis-Golgi compartments (Brandizzi and Barlowe, 2013; Barlowe and Helenius, 2016; Aridor, 2018; Hanna et al., 2018; Robinson et al., 2015). The COPII coat comprises two layers of protein complexes. The inner layer consists of a Sar1-Sec23-Sec24 lattice, while the outer layer is made up of Sec31-Sec13 heterotetramers (Stagg et al., 2008; Whittle and Schwartz, 2010; Bi et al., 2002; Fath et al., 2007). Sar1 is a small GTPase of the Ras super family that is the master regulator of COPII vesicle biogenesis (Nakano and Muramatsu, 1989). Assembly of COPII coat is initiated by the conversion of Sar1-GDP to Sar1GTP, which is mediated by Sec12, a guanine nucleotide exchange factor (GEF) that is an ER resident membrane protein (Barlowe and Schekman, 1993; Nakano and Muramatsu, 1989). At the ER surface, activated Sar1 recruits the Sec23Sec24 heterodimer, which associates with the ER membrane as an "inner coat". The Sar1-Sec23-Sec24-cargo "pre-budding" complex in turn recruits the Sec13Sec31 heterotetramer, referred to as an "outer coat" to the nascent bud (Aridor et al., 1998; Salama et al., 1993). The polymerization of Sec13-Sec31 heterotetramers induces membrane curvature and completes vesicle biogenesis (Fath et al., 2007; Stagg et al., 2006; Whittle and Schwartz, 2010).

Selective recruitment of cargos into COPII coated vesicles is driven by ER export signals located on cytoplasmically exposed regions of cargo proteins (Barlowe, 2003). ER export signals of most transmembrane cargo proteins are thought to interact directly with the inner coat components of COPII. However, some transmembrane and most soluble cargo proteins interact with COPII components through ER-resident transmembrane adaptors or receptors (Barlowe, 2003). Sec24 is the COPII component that has been implicated in recognizing these various sorting signals (Mossessova et al., 2003; Nufer, 2003). However, several studies suggested that Sec23 and Sar1 may also play roles in cargo sorting by directly binding to the export signals (Aridor et al., 2001; Mancias and Goldberg, 2007; Giraudo and Maccioni, 2003). 
Based on the high sequence similarity among COPII paralogs, and crossspecies complementation studies (De Craene et al., 2014; Khoriaty et al., 2018), it has been assumed that functions of COPII components are conserved across eukaryotes. While components of the COPII coat are well studied in budding yeast and mammalian cells, illustrating their molecular functions in plants has been more challenging. Comparative genomic analyses revealed that gene families encoding COPII components have undergone gene expansions in plants with Sec23 exhibiting the largest expansion (Aridor et al., 2001; Mancias and Goldberg, 2007; Giraudo and Maccioni, 2003; Schlacht and Dacks, 2015). While there are only one and two Sec23 genes in S. cerevisiae and mammals (eg. mice and humans) (Jensen and Schekman, 2011), respectively, there are seven in both P. patens and A. thaliana (Brandizzi, 2018). Since Sec23 and its heterodimeric partner Sec24 are implicated in cargo sorting, the larger Sec23 gene family could suggest that plants have more diverse sorting signals requiring specificity at the level of the Sec23/Sec24 heterodimer. Consistent with this, in contrast to yeast, which has a single gene for most COPII components, all other components of COPII in plants are encoded by multiple genes, suggesting that cargo specificity may translate to formation of distinct COPII complexes and vesicle populations. Alternatively, larger gene families could simply indicate that plants have higher functional redundancy in COPII encoded gene families. COPII components caused developmental defects. A complete knockout of AtSec24A was lethal (Faso et al., 2009; Nakano et al., 2009). However, a missense point mutation in AtSec24A (R693K) resulted in ER morphology defects and disruption of ER-Golgi integrity. Interestingly, these phenotypes could not be rescued by either AtSec24B or AtSec24C (Faso et al., 2009; Nakano et al., 2009),

111 suggesting that AtSec24B and C are functionally distinct from AtSec24A. Even 112 though AtSec24B and AtSec24C did not rescue the Atsec24a missense mutant 113 and each were not important for plant viability, they were shown to influence the 114 development of reproductive cells (Tanaka et al., 2013). Functional 115 diversification was also suggested by analysis of AtSec16 mutants in which 116 storage protein precursors were accumulated abnormally in Atsec16a null 117 mutants, but not in Atsec16b null mutants (Takagi et al., 2013). 
While $P$. patens and $A$. thaliana have similar numbers of genes encoding

119 COPII components, moss has several attributes that make it particularly suited to

120 study COPII function. P. patens juvenile tissues are haploid and comprise

121 multiple tissue types that establish the plant body. Protonemal tissue, which

122 emerges from the spore and is also readily propagated vegetatively by tissue

123 homogenization, is composed of tip-growing cells constituting a two-

124 dimensional filamentous network. The predictable patterns of growth and cell

125 division provide an easily tractable system for analysis of mutant phenotypes. As

126 a single-cell-layer, protonemal tissue also enables high-resolution microscopy of

127 endogenously tagged fluorescent proteins in live cells (Rensing et al., 2020). In

128 addition to amenable tissue morphology and haploid lifestyle, P. patens is

129 amenable to transient RNA interference for rapid identification of gene function

130 and exhibits high rates of CRISPR Cas9-mediated genome editing (Rensing et al.,

131 2020; Mallett et al., 2019). Here, we utilized these molecular genetic and live-cell

132 imaging tools to systematically characterize the Sec23 and Sec24 gene families.

133 Analysis of mutant phenotypes revealed a high degree of functional specificity

134 among Sec23 and Sec24 isoforms. Focusing on the Sec23 gene family, we

135 discovered that Sec23 isoforms differentially influence ER to Golgi trafficking,

136 and secretion to the plasma membrane as well as the size of presumptive ER exit 137 sites.

\section{Results}

\section{Sec23 and Sec24 isoforms differentially affect tip growth}

$141 \quad$ P. patens has seven Sec23 and seven Sec24 genes. Based on sequence

142 similarity, we found that there are four subclasses of Sec23 and Sec24 genes and

143 in both cases six of the seven genes exist in closely related pairs (Fig 1A, B). For

$144 \operatorname{Sec} 23$, with the exception of $\operatorname{Sec} 23 \mathrm{~A}$, at least one member of each subclass is

145 highly expressed in protonemal cells (Fig 1A). For Sec24, we found that one of

146 the seven Sec24 genes (Sec24E) was not expressed in protonemata (Fig1B). Of

147 the remaining six genes, Sec24D exhibited the lowest expression while the other

148 five Sec24 genes were expressed at similar levels (Fig 1B). To determine if Sec23

149 and Sec24 participate in protonemal growth, we used transient RNA interference

150 (RNAi) to silence the Sec23 and Sec24 gene families. We used a region of the 
151 coding sequence from a member of each subclass (Fig1A, B, solid lines under

152 gene models). These sequence regions share a high degree of sequence similarity to the other gene in each subclass (Fig 1A, B, dashed lines under gene models, Table S1) and would effectively simultaneously silence both genes. We did not target $\operatorname{Sec} 24 E$ since we were unable to detect a transcript.

By co-transforming the Sec23G RNAi construct together with a construct containing the target sequences for $\operatorname{Sec} 23 A, C$ and $D$, we targeted the entire $\operatorname{Sec} 23$ gene family and discovered that silencing the seven $\operatorname{Sec} 23$ genes leads to a dramatic reduction in growth (Fig 1C, D). Surprisingly, we found that silencing subclass III comprised of $\operatorname{Sec} 23 \mathrm{D}$ and $\operatorname{Sec} 23 \mathrm{E}$ resulted in a similarly severe growth defect (Fig 1C and D). In contrast, silencing any of the other subclasses did not affect growth (Fig 1C, D). These results demonstrated that there is functional specificity among Sec23 isoforms, with only subclass III playing a critical role in protonemal growth, while the remaining five Sec23 isoforms appear to be dispensable.

Similar to silencing subclass III Sec23 genes, silencing Sec24C and Sec24D resulted in a 79\% reduction in plant area (Fig 1E, F). Silencing Sec24F and Sec24G also impaired growth, but to a lesser extent resulting in a $48 \%$ reduction in plant area (Fig 1E, F). On the other hand, silencing Sec24A and Sec24B did not affect growth, although both genes were expressed at high levels. These data suggest that like Sec23, there is isoform specificity among Sec24 genes.

\section{Sec23 genes in subclass III are functionally redundant}

To test whether $\operatorname{Sec} 23 D$ and $\operatorname{Sec} 23 E$ within subclass III are functionally redundant, we designed RNAi constructs targeting the untranslated regions (UTRs) of each gene (Fig 2A). Targeting the untranslated regions of both Sec23D and $S e c 23 E$ resulted in small unpolarized plants with an $85 \%$ reduction in plant area as compared to control RNAi plants (Fig 2B, C). Silencing Sec23D alone resulted in a 59\% reduction in plant area, while silencing Sec23E did not affect plant area (Fig 2B, C). Given that Sec23D is expressed seven times higher than $\operatorname{Sec} 23 E$ (Fig 1B), it is possible that Sec23D contributes to protonemal growth more than Sec23E. To test whether Sec23E can replace Sec23D function if expressed at sufficient levels, we performed complementation studies by driving 
184

185

186

187

188

189

190

191

192

193

194

195

196

the full-length coding sequence of $\operatorname{Sec} 23 D$ or $\operatorname{Sec} 23 E$ with the constitutive maize ubiquitin promoter. Interestingly, the reduction in plant area resulting from silencing of $\operatorname{Sec} 23 D$ was restored to similar levels when either $\operatorname{Sec} 23 D$ or $\operatorname{Sec} 23 E$ full-length cDNAs were expressed (Fig 2B, C), indicating that Sec23D and Sec23E are functionally redundant, and that the highly expressed $\operatorname{Sec} 23 D$ is the predominant group III isoform critical for protonemal growth.

\section{CRISPR-Cas9-mediated deletion of Sec23 isoforms}

Using transient RNAi, we were able to deduce that Sec23 genes display isoform specific functions during protonemal growth. However transient RNAi assays are limited to phenotypic characterization of 7-day-old plants. To further characterize the molecular functions of Sec23, we generated stable loss-offunction mutants of Sec23 genes using CRISPR-Cas9 mediated genome editing. We targeted each gene with one protospacer located in the first coding exon (Fig S1A, S2). We obtained single and higher-order mutants of Sec23 genes carrying out-of-frame mutations resulting in premature stop codons in their transcripts (Fig S1A, S2, Table S2). Consistent with the RNAi results, 7-day-old $\Delta \sec 23 d$ plants were $70 \%$ smaller than wild type plants while $\Delta s e c 23 e$ plants exhibited no growth defects (Fig 3A, B). We isolated a number of sec23d null alleles (Fig S1A, Table S2), which had similar growth phenotypes (Figure S1B). We also found that in three-week-old plants regenerated from protoplasts, $\Delta \sec 23 d-5$ plants were $62.6 \%$ smaller than wild type (Fig 3C, D), and $\Delta s e c 23 d-5$ had fewer gametophores compared to wild type (Fig 3C, E), suggesting that slow growing protonemal tissues resulted in a developmental delay in the production of gametophores.

Although it was possible to isolate many $\Delta \sec 23 d$ and $\Delta \sec 23 e$ single mutants, we were unable to obtain $\Delta s e c 23 d e$ double mutants either by targeting $\operatorname{Sec} 23 D$ and $S e c 23 E$ simultaneously or by targeting $S e c 23 D$ in the $\Delta s e c 23 e-6$ single mutant background. This suggests that deleting both $\operatorname{Sec} 23 D$ and Sec23E is incompatible with protoplast regeneration, consistent with the severe growth defects observed by simultaneously silencing Sec23D and Sec23E using RNAi. Furthermore, we confirmed that none of the other Sec23 genes were upregulated 
216 in $\Delta s e c 23 d-5$ plants (Fig S1C), suggesting that the phenotypic consequences

217 observed were due specifically to loss of $\operatorname{Sec} 23 D$.

218 Since the RNAi results suggested that all other Sec23 subclasses were not

219 required for protonemal growth, we sought to isolate a stable line lacking

$220 \operatorname{Sec} 23 A, \operatorname{Sec} 23 B, \operatorname{Sec} 23 C, \operatorname{Sec} 23 F$, and $\operatorname{Sec} 23 G$ to determine if these genes

221 contribute to other aspects of plant growth and development. We successfully

222 isolated two independent quintuple mutant alleles $\Delta \sec 23 a b c f g-1$ and

$223 \Delta s e c 23 a b c f g-2$, in which Sec23A, Sec23B, Sec23C, Sec23F, and Sec23G contained

224 lesions resulting in null alleles for each gene (Fig S2, Table S2). Most lesions were

225 small deletions or insertions. However, $\Delta \sec 23 a b c f g-1$ contained a three

226 thousand base pair insertion in the Sec23G locus, still resulting in disruption of

227 the Sec23G locus (Fig S2, Table S2). We characterized both quintuple mutants

228 and found that while seven-day old $\Delta \sec 23 a b c f g-1$ plants exhibited no detectable

229 growth defects, $\Delta s e c 23 a b c f g-2$ plants exhibited a mild growth defect (Fig 3B, C).

230 However, three-week-old $\Delta s e c 23 a b c f g-1$ plants were larger than wild type, and

$231 \Delta s e c 23 a b c f g-2$ plants were indistinguishable from wild type (Fig 3D, E).

232 Interestingly, both $\Delta s e c 23 a b c f g-1$ and $\Delta s e c 23 a b c f g-2$ developed more

233 gametophores than wild type (Fig 3E), suggesting that these five Sec23 genes

234 may negatively regulate gametophore development.

235 Even though the two quintuple mutant isolates, exhibited statistically

236 significant differences at different times in development between each other and

237 wild type, these were minor compared to the differences between wild type and

238 all isolated $\Delta s e c 23 d$ null mutants. To investigate whether the observed

239 differences might result from differential upregulation of Sec23D or Sec23E in the

$240 \Delta s e c 23 a b c f g$ mutants thereby compensating for the loss of Sec23ABCFG

241 functions, we measured the expression levels of $S e c 23 D$ and $S e c 23 E$ transcripts

242 in $\Delta s e c 23 a b c f g-1, \Delta \sec 23 a b c f g-2$, and wild type. In 8-day-old plants, we found

243 that neither Sec23D nor Sec23E transcripts were elevated compared to

244 expression in wild type (Fig 3F), indicating that minor differences observed

245 between the two quintuple mutants were not readily explained by increased

246 expression of either Sec23D or E. Taken together, the phenotypic analyses of

247 stable $\sec 23$ null plants suggest that $\operatorname{Sec} 23 A, B, C, F$ and $G$ do not contribute 
248 substantially to establishment of juvenile moss tissues under laboratory growth conditions. In contrast, $\operatorname{Sec} 23 D$ and $E$ are essential.

\section{Loss of Sec23D causes ER morphology defects and ER stress}

As a predicted member of the COPII complex, Sec23D likely mediates ERto-Golgi transport. However, it is unclear whether the $\Delta s e c 23 d$ growth defect is a result of a general impairment of ER-to-Golgi transport, or the lack of specific cargo sorted by Sec23D needed for protonemal growth. Studies in other organisms have shown that blocking COPII function alters ER morphology (Novick et al., 1980) and results in elevated levels of unfolded proteins in the ER, leading to activation of the ER unfolded protein response (Belden, 2001; Chung et al., 2016). To analyze Sec23D's function in ER-to-Golgi transport, we investigated whether loss of Sec23D affects ER morphology and the ER unfolded protein response. We used CRISPR-Cas9 mediated genome editing to knock out Sec23D in a line where the ER is fluorescently labeled by targeting GFP to the ER lumen (Fig S1, Table S2). When imaged with a confocal microscope, $\Delta \sec 23 d$-B11 cells exhibited numerous ER dots (Fig 4A, red and cyan boxes), and 65\% of protonemal filaments contained large ER aggregates (Fig 4A, green and yellow boxes). Imaging with a super resolution spinning disc confocal system allowed closer inspection of the aggregates (Fig. 4B). Smaller aggregates were clearly comprised of accumulated tubules, while large aggregates appeared to have tubules that wrapped around each other (Fig. 4B, Movie S1).

If changes in ER morphology result from defective secretion at ER exit sites, then proteins may be inadvertently accumulating in the ER, resulting in upregulation of the unfolded protein response. To test this, we analyzed the expression of an ER heat shock protein (Bip), which is a well-known ER stress

274 reporter in Arabidopsis thaliana (Noh et al., 2003; Srivastava et al., 2013;

275 Maruyama et al., 2014; Cho and Kanehara, 2017). P. patens has two Bip paralogs,

276 Bip1 and Bip2. Since Bip2 expression is low in protonemal tissue (Ortiz-Ramírez 277 et al., 2017), we tested whether Bip1 is affected under conditions that induce ER 278 stress. Brefeldin A (BFA) inhibits protein secretion by disrupting ER-Golgi 279 integrity (Nebenfuhr, 2002; Niu et al., 2005). We found that after a 24-hour 280 treatment of BFA, expression of Bip1 increased by about two-fold (Fig 4C), 
281 indicating that Bip1 is a plausible ER stress reporter in P. patens. Interestingly,

282

283

284

285

286

287

288

289

290

291

292

293

294

295

296

297

298

299

300

301

302

303

304

305

306

307

308

309

310

311

312

313

Bip1 expression increased 5-fold in $\Delta s e c 23 d$ compared to in wild type (Fig 4D),

demonstrating that $\Delta s e c 23 d$ suffers from severe ER stress. In contrast, $\Delta \sec 23 a b c f g-1$, which didn't exhibit significant growth defects, had normal levels of Bip1 expression (Fig 4D), and normal ER morphology (Fig 4A).

\section{Disrupting Sec23D results in reduced ER to Golgi trafficking and secretion to} the plasma membrane

ER morphology defects and elevated ER stress in $\Delta s e c 23 d$ suggest that transport of secretory cargos might be blocked. We reasoned that proteins destined to the Golgi and the plasma membrane would accumulate in the ER in mutants with impaired ER to Golgi transport. To test this, we disrupted Sec23D in lines that either express YFP fused to the first 49 amino acids of the soybean

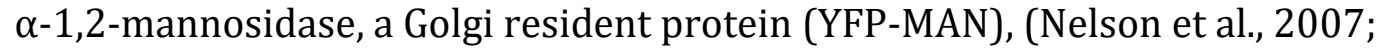
Furt et al., 2012) or an mCherry fusion of a transmembrane protein (F-SNAPmCherry) previously used to measure exocytosis (van Gisbergen et al., 2018) (Fig S1, Table S2). Since it would have been time-consuming to regenerate quintuple mutants in these two backgrounds, we instead transformed the YFPMAN and F-SNAP-mCherry markers into $\Delta s e c 23 a b c f g-1$ plants. We selected lines that had similar expression levels to control lines by measuring the total amount of fluorescence in cells.

In control YFP-MAN lines, YFP signal accumulated in the Golgi with very little fluorescence observed in the rest of the cell (Fig 5A). In contrast in $\Delta \sec 23 d$ F12, while YFP fluorescence still accumulated in the Golgi, a large portion of the fluorescent signal that was presumably retained in the ER was observed diffusely throughout the cell (Fig 5A). To quantify ER to Golgi trafficking efficiency, we calculated the fluorescence intensity ratio of the signal in the Golgi divided by the signal in the cytoplasm. A high ratio value indicates efficient delivery to the Golgi, as observed in the control lines (Fig 5B). In contrast, $\Delta s e c 23 d-F 12$ exhibited a significantly lower fluorescence intensity ratio (Fig 5B), indicating that loss of Sec23D impairs delivery of YFP-MAN to the Golgi. Imaging of $\Delta s e c 23 a b c f g-1$ YFP-MAN and its control line revealed that the majority of the YFP signal localizes to the Golgi with the Golgi to cytoplasm ratios in 
$314 \Delta s e c 23 a b c f g-1$ indistinguishable from the control (Fig 5B), suggesting that

315 trafficking of YFP-MAN to the Golgi is not affected in the quintuple mutant.

316 Many proteins transported in COPII-coated vesicles ultimately reside at the

317 plasma membrane, are secreted, or are trafficked to the vacuole. Without a

318 fluorescent vacuolar cargo in hand, we instead investigated vacuolar

319 morphology by staining the vacuole with MDY-64, a dye that stains the tonoplast

320 in living plant cells (Scheuring et al., 2015). While we found that vacuolar

321 morphology was similar between wild type, $\Delta s e c 23 d-5$ and $\Delta s e c 23 a b c f g-1$ plants

322 (Fig S3), we can not rule out that vacuolar protein sorting has not been affected.

To investigate trafficking to the plasma membrane, we used an engineered fluorescent protein cargo, F-SNAP-mCherry, previously used to analyze exocytosis in protonemata (van Gisbergen et al., 2018). In wild type cells, the FSNAP-mCherry accumulated on the plasma membrane, with only a very small amount of fluorescence detected inside the cell (Fig 5C). In $\Delta s e c 23 d-S 2$, a large portion of the mCherry signal was retained in the cell, labeling the ER network (Fig 5C). To quantify secretion efficiency to the plasma membrane, we calculated the fluorescence intensity ratio of the plasma membrane to the intensity within the cell. We found that the ratio in $\Delta s e c 23 d-S 2$ was significantly lower than that of its control line (Fig 5D), indicating that loss of Sec23D inhibits secretion of plasma membrane proteins.

Interestingly, we found that the F-SNAP-mCherry signal was also retained inside the cell in the $\Delta s e c 23 a b c f g-1$ mutant, readily observing labeling of the ER network (Fig 5C). In addition, the fluorescence intensity ratio in $\Delta s e c 23 a b c f g-1$ was lower in comparison to the ratio in its control line (Fig 5D). However, the ratio in $\Delta s e c 23 a b c f g-1$ was still significantly higher than the ratio in $\Delta s e c 23 d-S 2$, suggesting that secretion of plasma membrane proteins is impaired in $\Delta \sec 23 a b c f g-1$ but to a lower extent than in $\Delta s e c 23 d-S 2$. These data together with

341 the lack of a growth defect in protonomata in the $\Delta s e c 23 a b c f g-1$ mutant suggest

342 that Sec23A, B, C, F, and G contribute to protein secretion particularly to the 343 plasma membrane, but importantly their cargos are not essential for polarized 344 growth in protonemata. 


\section{6}

347

348

349

350

351

\section{Loss of Sec23D does not affect the cytoskeleton during tip growth}

To investigate if defects in protein secretion, resulted in alterations to the cytoskeleton, critical for polarized expansion in protonemata, we imaged microtubules and actin in wild type and in cells carrying null mutations in Sec23D. Microtubules align along the length of the cell with their plus ends focused just below the cell apex (Hiwatashi et al., 2014), where a dynamic cluster of actin filaments forms predicting the site of cell expansion (Wu et al., 2018). Wild type and $\Delta s e c 23 d-M 4$ cells expressing GFP-tubulin exhibit indistinguishable microtubule networks and both have a focal point of microtubules near the cell tip (Fig S4A, arrows). Using lifeact-mCherry to label the actin network, we found that both wild type and $\Delta \sec 23 d-5$ cells form an apical actin cluster that associates with the growing tip (Fig S4B). Time-lapse imaging revealed no discernable differences in the dynamics of the apical actin cluster between wild type and $\Delta \sec 23 d-5$ (Fig S4C, Movie S2). These data suggest that tip growth defects in cells lacking Sec23D are independent of the cytoskeleton.

\section{Sec23 isoforms localize to discrete puncta associated with the ER}

If Sec23 is a bona fide COPII component, then we would expect that Sec23 isoforms should associate with the ER. To test this, we inserted sequences in the genome of a line expressing GFP in the ER lumen encoding for three tandem mRuby2 (hereafter, 3XmRuby) in frame with the coding sequences of the three mostly highly expressed protonemal Sec23 genes: $\operatorname{Sec} 23 B, \operatorname{Sec} 23 D$ and $\operatorname{Sec} 23 G$ (Fig 1B, Fig S5A, B). To ensure that tagging Sec23D did not affect its function, we measured plant area from 7-day old regenerated protoplasts and found that Sec23D-3XmRuby grew indistinguishably from the control lines (Fig S5C), suggesting that Sec23D-mRuby is functional. By analogy, we reasoned that tagging $\operatorname{Sec} 23 B$ and $G$ would also be functional. To ensure we did not observe any possible dominant negative impacts as a result of the tagging, we performed growth assays of 7-day old plants regenerated from protoplasts and observed that Sec23B-3XmRuby was indistinguishable from the control and Sec23G-mRuby was slightly smaller (Fig S5C). 

Sec23G localized to punctate structures throughout the cytoplasm (Fig 6A). All three proteins labeled puncta that were often proximal to the ER (Fig 6A). Colocalization measurements using Pearson's Correlation Coefficient suggested that Sec23D, B and G puncta positively correlated with the ER. To ensure that this correlation was significant, we flipped the ER image both vertically and horizontally and repeated the correlation analysis and found that the correlation coefficients were significantly lower (Fig 6B). Interestingly, Sec23G-3XmRuby puncta were larger than the punctate structures found in Sec23D- and Sec23B3XmRuby lines (Fig 6A, C). To quantify possible size differences, we imaged all three tagged Sec23 isoforms with a pixel size that maximizes the resolving capacity of the imaging system (Fig 6C, see Materials and Methods) and utilized a segmentation tool in Fiji that identifies particles in the images (Gilles et al., 2017). We discovered that Sec23G particles are significantly larger than Sec23B and D. Furthermore, Sec23D is slightly larger than Sec23B. These data suggest that Sec23 isoforms form distinct compartments on the ER.

\section{Sec23B and Sec23G localization is independent of Sec23D.}

To further probe the nature of the Sec23 particles, we analyzed the localization of Sec23B and Sec23G with respect to Sec23D. We used CRISPR-Cas9 mediated homology directed repair (HDR) to insert sequences encoding for mNeonGreen (hereafter, mNeon) in-frame with Sec23B or Sec23G in a Sec23D

400 line endogenously tagged with a single mRuby2 (hereafter, mRuby) (Fig S6). As 401 expected Sec23B-mNeon formed small puncta whereas Sec23G-mNeon formed 402 larger puncta (Fig 7A, B). Imaging of Sec23B-mNeon and Sec23D-mRuby 403 revealed the presence of a distribution of cytoplasmic puncta, some with only 404 Sec23B-mNeon or only Sec23D-mRuby and some with both (Fig 7A). In contrast, 405 Sec23G-mNeon and Sec23D-mRuby were largely non-overlapping (Fig 7B). To 406 quantify the degree of overlap, we utilized the segmentation tool in Fiji that 407 identifies puncta and then determines the number of segmented particles that 408 physically overlap (Gilles et al., 2017). We found that $57 \%$ of Sec23B-mNeon co409 localized with Sec23-mRuby. In contrast, only 4\% of Sec23G-mNeon showed 410 overlap with Sec23-mRuby (Fig 7C). In addition to identifying co-localizing 
411 particles, the tool also measures the center-to-center distance of overlapping

412 particles (Gilles et al., 2017). Sec23G-mNeon and Sec23-mRuby had a

413 significantly larger center-to-center distance as compared to Sec23B-mNeon and

414 Sec23D-mRuby (Fig 7D), suggesting that any overlap between Sec23G and

415 Sec23D is coincidental.

416 While Sec23B and Sec23D exhibited a high degree of co-localization,

417 COPII vesicles are significantly smaller than the limit of resolution of a light

418 microscope. Therefore, it is unclear if a single vesicle contains both Sec23B and

419 Sec23D or if vesicles form with a single Sec23 isoform. Given that Sec23G

420 structures are distinct from Sec23D, and 40\% of Sec23B and Sec23D puncta are

421 not overlapping, we hypothesized that Sec23B, Sec23D and Sec23G form distinct

422 ER exit sites. If this is the case, we would expect that Sec23B and Sec23G would

423 be unaffected by loss of Sec23D. Indeed, we discovered that when we deleted

424 Sec23D in the Sec23B-3XmRuby and Sec23G-3XmRuby lines, both Sec23B and

425 Sec23G still localized to puncta (Fig 7E). We quantified the size of the puncta and

426 found that Sec23G-3XmRuby was unaffected but Sec23B-3XmRuby were slightly

427 larger in the absence of Sec23D (Fig 7F). However, given that the average

428 Sec23B-3XmRuby particle size is essentially at the limit of resolution of our

429 system, it is unclear how significant this increase may be. Nevertheless, these

430 results demonstrate that even when ER morphology is affected due to loss of

431 Sec23D, Sec23B and Sec23G are largely unaffected, suggesting that Sec23B and

432 Sec23G ER exit sites are independent of Sec23D.

\section{Discussion}

435 The large number of genes encoding components of COPII in plants 436 together with functional studies in Arabidopsis (De Craene et al., 2014; Chung et 437 al., 2016; Barlow and Dacks, 2018; Brandizzi, 2018; Faso et al., 2009; Qu et al., 438 2017; Tanaka et al., 2013) have led to the hypothesis that gene expansion 439 underlies increased functional diversification required for complex secretory 440 processes that pattern and build plant cells. Evidence suggests that functional 441 diversification could stem from tissue/developmental specific expression (Hino 442 et al., 2011; Lee et al.; Hanton et al., 2009) or from the formation of COPII 443 complexes comprised of specific isoforms with unique functions (Zeng et al., 
444 2015; Zeng et al., 2021). However, it remains unclear whether unique COPII

445 complexes mediate transport of specific cargos or accumulate at some ER exit

446 sites and not at others. Here, by analyzing the role of Sec23 and Sec24 isoforms

447 in protonemal development, we have provided evidence for formation of distinct

448 ER exit sites and the requirement of only Sec23D/E for transport of cargos

449 uniquely required for tip growth.

450 Using RNAi, we showed that Sec23 and Sec24 isoforms differentially

451 impact protonemal growth. Silencing of Sec23D/E or Sec24C/D similarly inhibit

452 tip growth suggesting that $S e c 23 D / E$ may interact with $S e c 24 C / D$. However,

$453 \mathrm{Sec} 24 \mathrm{~F} / G$ also exhibited reduced growth. Thus, it is possible that a number of

454 heterodimer pairs may form that are critical for polarized growth. Protein-

455 protein interactions studies are needed to confirm if isoform specific

456 heterodimers form in moss. If this is the case, it suggests that isoform specificity

457 regulates COPII function similar to the finding in Arabidopsis that Sar1A and

458 Sec23A form a unique interaction mediating functional specificity (Zeng et al.,

459 2015).

460 Focusing on Sec23, where one of the Sec23 pairs, Sec23D/E, plays an

461 essential role in protonemal growth, we found several lines of evidence

462 suggesting that Sec23D functions as a bona fide COPII component. First, $\Delta \sec 23 d$

463 plants had abnormal ER morphology and elevated ER stress consistent with a

464 block in cargo secretion from the ER. Second, trafficking to the Golgi and

465 secretion to the plasma membrane was reduced in $\Delta s e c 23 d$. Third, Sec23D

466 localized to puncta near the ER. Due to differential expression levels, we found

467 that Sec23D is more important for protonemal growth than Sec23E. However,

468 when expressed at sufficient levels, Sec23E can rescue loss of Sec23D indicating

469 that Sec23D and Sec23E are functionally redundant. The inability to recover a

470 double mutant lacking both Sec23D and E suggests that together these two genes

471 are essential for moss viability. Given that viability in moss is measured in the

472 ability to generate protonemata, which is a tip-growing tissue, we reason that

473 Sec23D and E are essential for tip growth. While $\Delta s e c 23 d$ protonemal cells grow

474 significantly slower than wild type leading to smaller plants, we were unable to

475 identify any defects in either the actin or microtubules cytoskeletons, suggesting

476 that Sec23D contributes to polarized cell expansion independent of the 
cytoskeleton. In fact, tip growth in $\Delta s e c 23 d$ resembles the recently identified $P$. patens $\Delta$ sabre mutant plants (Cheng \& Bezanilla, 2021). SABRE associates with regions of the ER and its absence has no effect on the cytoskeleton but rather impacts ER morphology by accumulating aggregates that label with an ER lumenal marker (Cheng \& Bezanilla, 2021). Taken together these data suggest that abrogated ER function impacts polarized growth without altering cytoskeletal organization and dynamics.

With respect to gametophore formation, $\Delta s e c 23 d$ develops gametophores late, but once gametophores form they are morphologically normal. This suggests that Sec23D function is specifically required during tip growth. In contrast, we found that the remaining five $\operatorname{Sec} 23$ genes were dispensable for formation of both protonemata and gametophores. One possible explanation for this finding is that Sec23A, B, C, F and G have functionally diversified no longer playing a role in ER to Golgi transport. In support of this hypothesis, unlike $\Delta s e c 23 d, \Delta s e c 23 a b c f g$ plants did not exhibit ER morphology defects or increased ER stress characteristic of cargo backing up in the ER often observed with COPII loss of function mutants. However, we did observe secretion defects of plasma membrane cargo in $\Delta s e c 23 a b c f g$ plants. Given that Sec23B and G localize proximal to the ER independent of Sec23D, it is possible that Sec23B and G contribute to COPII function via pathways that may potentially bypass the Golgi (Cheng et al., 2009; Zhang et al., 2011; Ding et al., 2012; Wang et al., 2017). Alternatively, Sec23B and G may also function in ER to Golgi trafficking but, in contrast to Sec23D, their cargos may be fewer and may not be essential for tip growth.

Interestingly all the mutant combinations that we generated formed morphologically normal gametophores. However, since we were unable to generate $\Delta s e c 23 d e$ or $\Delta s e c 23 a b c e f g$ plants, we can not distinguish between

504 Sec23E playing a critical role in gametophore formation versus Sec23D or

505 Sec23E acting redundantly with Sec23A, B, C, F and G for COPII secretion 506 required to shape gametophores. If, on the other hand, COPII is not required for 507 gametophore formation, then future experiments conditionally removing $508 \mathrm{Sec} 23 \mathrm{D} / \mathrm{E}$ in $\Delta s e c 23 a b c f g$ plants would be expected to form normal 509 gametophores and would be an excellent tool to identify COPII-independent 
510

511

512

513

514

515

516

517

518

519

520

521

522

523

524

525

526

527

528

529

530

531

532

533

534

535

536

537

538

539

540

541

542

secretion pathways in plants, which are thought to play a role in transport of tonoplast resident proteins such as the vacuolar $\mathrm{H}^{+}$-ATPase, VHA-a3 (Viotti et al., 2013).

While Sec23B, Sec23D, and Sec23G formed puncta associated with the ER, these puncta were distinct. Sec23G formed large dots near the ER, whereas Sec23D and Sec23B were smaller. Sec23B and Sec23D puncta exhibited a high degree of overlap, suggesting that Sec23B and Sec23D may form vesicles in the same region of the ER. In contrast Sec23G largely did not overlap with Sec23D and by extension likely not Sec23B. Formation of larger domains on the ER suggests that Sec23G may be involved in the secretion of bigger cargo and provides evidence that moss has morphologically distinct ER exit sites, that can form, even when ER morphology is disrupted, independent of Sec23D.

Given that Sec23D contributes to ER to Golgi trafficking and secretion to the plasma membrane, while Sec23A, B, C, F and G contributes to plasma membrane secretion, it is plausible to suggest that tip growth requires intact ER to Golgi trafficking. In $\Delta s e c 23 d$ plants cargos essential for tip growth are either inefficiently carried from the ER to the Golgi or are not properly processed in the Golgi due to severe reductions in trafficking to the Golgi. Perhaps there is a larger burden for carbohydrates synthesized by enzymes in the Golgi to generate a tip growing cell wall as compared to a diffusely growing cell wall. To test this, future studies will focus on identifying cargos that have been differentially affected in $\Delta s e c 23 d$ versus $\Delta s e c 23 a b c f g$ plants. Comparative mass spectrometry of isolated microsomes and plasma membrane extracts in these mutant backgrounds could be very informative. In addition, quantitatively measuring secretion of known Golgi resident carbohydrate biosynthetic enzymes and plasma membrane complexes such as cellulose and callose synthases in these mutants could help to narrow down cargo specificity.

Taking advantage of the facile molecular genetic manipulation and imaging in moss protonemal development, our study has shown that the expanded Sec23 gene family in moss is multi-faceted. We found evidence that the Sec23 gene family exhibits aspects of functional redundancy, differential gene expression, as well as isoform specificity for ER exit site formation, ER to Golgi trafficking, and importantly transport of essential cargos required for tip growth. 
bioRxiv preprint doi: https://doi.org/10.1101/2020.06.22.165100; this version posted June 5, 2021. The copyright holder for this preprint (which was not certified by peer review) is the author/funder. All rights reserved. No reuse allowed without permission.

543 Building upon the tools developed in this study, future studies will be able to

544 characterize the remaining COPII components, thereby providing a complete

545 picture of how gene expansions in COPII have been deployed. 


\section{Materials and Methods}

\section{Expression analyses}

To quantify expression of the $\operatorname{Sec} 23$ and $\operatorname{Sec} 24$ genes family members (Table S3), as well as Bip1 (Pp3c10_17310), we isolated total RNA from 8-day old plants (various genotypes, as needed for the particular experiment) regenerated

551 from protoplasts using the RNeasy Plant mini prep kit (Qiagen). cDNA was prepared from $1 \mu \mathrm{g}$ of total RNA using SuperScriptIII reverse transcriptase kit, following the manufacturer's recommendation (Invitrogen). qPCR assays were conducted with Luna Universal qPCR Master Mix (New England Biolabs).

555 Primers for qRT-PCR analyses are listed in Table S4. After confirming that amplification efficiencies (calculated from standard curves) were similar for each set of primers (Table S4), relative expression levels were calculated, and expression of Ubiquitin 10 was used for normalization. All experiments had three technical replicates and three biological replicates.

\section{Constructs}

The Sec23 and Sec24 RNAi constructs were generated by PCR amplification of either the coding sequence or 5' and 3' untranslated region

564 (UTR) of Sec23 or Sec24 from P. patens cDNA using primers indicated in Table S4.

565 PCR fragments were cloned into pENTR-D-TOPO (Invitrogen) following the manufacturer's recommendations and the resulting vectors were sequenced. The Sec23ACD-RNAi and Sec23 UTR-RNAi constructs were generated by stitching together multiple PCR fragments and then directionally cloned into pENTR-DTOPO as previously described (Vidali et al., 2007). LR clonase (Invitrogen) reactions were used to transfer either the coding sequence or 5' and 3' UTR sequences the RNAi vector pUGGi (Bezanilla et al., 2005) generating the Sec23

572 UTRi or Sec24-CDSi constructs, respectively. Restriction enzyme digestion was 573 used to verify these constructs.

Expression constructs were generated by PCR amplifying Sec23D and

575 Sec23E coding sequences from $P$. patens cDNA using a 5' CACC sequence on the 576 specific primers for oriented cloning into the pENTR-D-TOPO vector

577 (Invitrogen). LR clonase (Invitrogen) reactions were used to transfer these 578 coding sequence into pTH-Ubi-Gate vectors (Vidali et al., 2007). 

sequences upstream and downstream of the stop codon for $\operatorname{Sec} 23 D, \operatorname{Sec} 23 B$, and Sec23G using the primers in Table S4. For Sec23D, we used homologous recombination. The distance between the 5' and 3' homology arms was 500bp,

583 which when inserted correctly the tagging construct replaces these 500bp with sequences encoding for the fluorescent protein and an antibiotic resistance cassette (Fig S5, Fig S6). Using BP clonase (Invitrogen), homology arms were transferred to vectors, generating homology arm entry clones. Four-fragment recombination reactions were used to assemble the homology arms, fluorescent protein sequence, and the antibiotic resistance cassette into pGEM-gate (Vidali et al., 2009). For Sec23B and Sec23G tagging, we used CRISPR Cas9-mediated HDR. 5' and 3' homology arms upstream and downstream of the stop codon, with a distance between these two arms of 38bp for Sec23B and 131bp for Sec23G were 592 PCR amplified from genomic DNA (Fig S5, Fig S6). Using BP clonase (Invitrogen),

593 homology arms were transferred to vectors, generating homology arm entry 594 clones, as described in (Mallett et al., 2019). Three-fragment recombination reactions were used to assemble the homology arms and the fluorescent protein

596 sequence into pGEM-gate. We chose the CRISPR-Cas9 protospacer close to the 597 stop codon.

Protospacers for CRISPR-Cas9 mediated mutagenesis and HDR were 599 designed with the CRISPOR online software (crispor.tefor.net) (Haeussler et al., 600 2016). Protospacers were selected with high specificity scores and low off-target 601 frequency. Specific overhangs sequences were added to facilitate downstream 602 cloning. Protospacers were synthesized as oligonucleotides (Table S4) and 603 annealed as described (Mallett et al., 2019). Annealed protospacer fragments 604 were transferred to vectors using Instant Sticky-End Ligation Master Mix (New 605 England Biolabs). Sec23B and $G$ protospacers designed to tag these genes via $606 \mathrm{HDR}$ as well as $\operatorname{Sec} 23 A, D$ and $E$ protospacers designed to knock out these genes 607 were ligated into pENTR-PpU6-sgRNA-L1L2 (Mallett et al., 2019). Sec23B and F 608 protospacers designed to knock out these genes were ligated into pENTR-PpU6609 sgRNA-L1L5 (Mallett et al., 2019). Sec23C and G protospacers designed to knock 610 out these genes were ligated into pENTR-PpU6-sgRNA-R5L2 (Mallett et al., 611 2019). LR clonase (Invitrogen) was used to assemble the protospacer containing 
612 vectors into pZeo-Cas9-Gate, pMH-Cas9-Gate, or pMK-Cas9-Gate vectors (Mallett

613 et al., 2019), as needed. Two-fragment recombination reactions were used to

614 assemble dual targeting mutagenesis vectors for $\operatorname{Sec} 23 B$ and $C$, and for $\operatorname{Sec} 23 F$

615 and $G$. Restriction enzyme digestion and sequencing was used to verify these

616 constructs.

617

\section{Tissue propagation, transformation, and genotyping}

619 All moss lines were propagated weekly by moderate homogenization in

620 water and pipetting onto a permeable cellophane covered solid media, as

621 described previously (Wu and Bezanilla, 2014). Tissue was grown at room

622 temperature in Percival growth chambers, under $85 \mu \mathrm{mol}_{\text {photons }} / \mathrm{m}^{2} \mathrm{~s}$ light with

623 long-day conditions. PEG-mediated transformations were performed as

624 previously described to generate stable lines (Wu and Bezanilla, 2014), CRISPR-

625 Cas9 genome edited lines (Mallett et al., 2019) and perform transient RNAi

626 (Vidali et al., 2007).

To genotype mutants generated by CRISPR-Cas 9 mutagenesis, we

extracted DNA from plants that were 3-4 weeks old (0.5-1 cm in diameter) using

629 the protocol described in (Augustine et al., 2011). For editing experiments, we

630 used primers (Table S4) surrounding the expected Cas9 cleavage site $(\sim 300-400$

631 bp on each side). T7 endonuclease assay was used to screen candidate mutants

632 as described (Mallett et al., 2019). For homology-directed repair tagging and

633 homologous recombination tagging, we used primers (Table S4) outside of the

634 homology region to avoid amplification of residual DNA donor template.

\section{Imaging and morphometric analysis of growth assays}

For transient RNAi, complementation analyses, and growth assays of stable lines, plants regenerated from protoplasts were photographed seven days

639 after protoplasting. For transient RNAi and complementation analyses plants

640 lacking nuclear GFP signal were imaged because these are the plants that are

641 actively silencing as described previously (Bezanilla et al., 2005; Vidali et al.,

642 2007). For growth assays of stable lines any regenerated plant was imaged.

643 Images were acquired with a $1 \mathrm{X}$ objective using a stereomicroscope (Leica

644 MZ16FA or Nikon SMZ25) equipped with a CCD camera (Leica DF300FX or 
645 Nikon digital sight DS-Fi2). Chlorophyll fluorescence and any nuclear GFP signal were acquired simultaneously using filters (480/40, dichroic 505 (Leica) or

647 dichroic 510 (Nikon), emission 510 long pass). Exposure settings were

648 maintained constant throughout an experiment. 3-week old plants regenerated

649 from protoplasts were imaged with white light on a Nikon SMZ25 stereomicroscope equipped with a Nikon digital sight DS-Fi2 color camera.

To quantify plant area, individual plants were cropped from images and the red channel, representing chlorophyll fluorescence of the plant, was separated from the RGB image. Threshold settings were set manually for all

654 plants within an experiment. To quantify brightfield images of 3-week-old plants, the blue channel, representing chlorophyll fluorescence of the plant, was separated from the RGB image and thresholded. Total area was calculated from the largest thresholded object in the selected window. All images analysis was done using macros (Galotto et al., 2019) written for ImageJ. Analysis of variation (ANOVA) for multiple comparisons was performed with Kaleidagraph (Synergy) using the Tukey HSD post hoc tests. The alpha for statistical significance was set to 0.05 .

662

\section{Laser scanning confocal microscope imaging and quantification}

For imaging Sec23B/D/G localization and dynamics, 5 to 8-day old plants regenerated from protoplasts were placed onto an agar pad in Hoagland's buffer

$666\left(4 \mathrm{mM} \mathrm{KNO}_{3}, 2 \mathrm{mM} \mathrm{KH}_{2} \mathrm{PO}_{4}, 1 \mathrm{mM} \mathrm{Ca}\left(\mathrm{NO}_{3}\right)_{2}, 89 \mu \mathrm{M}\right.$ Fe citrate, $300 \mu \mathrm{M} \mathrm{MgSO}_{4}$, 9.93 $\mu \mathrm{M} \mathrm{H}_{3} \mathrm{BO}_{3}, 220 \mathrm{nM} \mathrm{CuSO}_{4}, 1.966 \mu \mathrm{M} \mathrm{MnCl}_{2}, 231 \mathrm{nM} \mathrm{CoCl}, 191 \mathrm{nM} \mathrm{ZnSO} 4$, $169 \mathrm{nM} \mathrm{KI}, 103 \mathrm{nM} \mathrm{Na} \mathrm{MoO}_{4}$, and $1 \%$ sucrose), covered by a glass cover slip and sealed with VALAP (1:1:1 parts of Vaseline, lanoline, and paraffin). For imaging ER morphology, Golgi resident protein secretion, F-SNAP-mCherry secretion, we used microfluidic imaging chambers (Bascom et al., 2016). Ground protonemal

672 tissue was gently pipetted into the central part of the device followed by an 673 infusion of Hoagland's medium. Then the chamber was submerged Hoagland's medium, and placed under constant $85 \mu \mathrm{mol}_{\text {photons }} / \mathrm{m}^{2} \mathrm{~s}$ light. 
678

679

680

681

682

683

684

685

686

687

688

689

690

691

692

693

694

695

696

697

698

699

700

701

702

703

704

705

706

707

708

709

710

illumination at $488 \mathrm{~nm}$ was used for exciting mNeon, YFP, GFP and chlorophyll autofluorescence; $561 \mathrm{~nm}$ for mRuby, mCherry. Emission filters were 525/50 nm for mNeon, YFP, and GFP; 595/50 nm for mRuby and mCherry.

Super resolution spinning disc confocal imaging was performed with a Nikon CSUW1-SoRA system. Images were acquired with a 60x Plan Apo 1.40NA objective with the $2.8 \mathrm{x}$ mag lens. Laser illumination at $488 \mathrm{~nm}$ was used for exciting GFP, and the Emission filter were 525/50. Images were denoised using the denoise.ai machine learning algorithm and deconvolved using the Richardson-Lucy algorithm within the NIS-Elements v5.21 software.

For quantification of trafficking to the Golgi, confocal Z-stacks of individual cells were converted to a maximum intensity projection. For quantification of secretion to the plasma membrane, the medial focal plane was selected for analysis. YFP (YFP-MAN) and mCherry (FSnap-mCherry) fluorescence intensities with the same expression pattern were normalized using enhanced contrast in Fiji. Images were cropped to isolate regions only within the cell. Then, using a minimum threshold of 20,000-22,000 it was possible to isolate and measure the fluorescence intensity of the Golgi structures (Golgi intensity) or the plasma membrane with analyze particles in Fiji. The fluorescence intensity of the background was similarly measured using the same threshold cut-off for each image but unchecking dark background, thereby selecting all regions below the threshold cut off. These values were used to calculate the ratio of Golgi/plasma membrane intensity to background intensity. To quantify the Pearson's Correlation Coefficient, we analyzed single focal planes with the Coloc2 plugin in Fiji comparing the original images as well as horizontally and vertically flipped ER-GFP images. To quantify the size and colocalization of Sec23B, D and G dots, we analyzed single focal planes and used the diAna tool for ImageJ, which uses object-based identification for distance and colocalization measurements (Gilles et al., 2017) and was recently used to define specific subdomains of the trans Golgi network in plant cells (Heinze et al., 2020). All images were acquired with a pixel size of $0.07 \mu \mathrm{m}$ and processed by subtracting background, enhancing contrast with normalization, and smoothing in Fiji prior to performing segmentation with diAna. For Sec23G-mNeon, chloroplast autofluorescence was collected in the far-red channel and subtracted 
711 from the green channel. To segment the dots in the image, we used the iterative

712 option with a minimum threshold of $25,000-27,500$ and a step value of 20 . After

713 segmentation, the diAna analyse module was used to measure the size of all

714 segmented regions of an image, as well as to determine the percent of co-

715 localizing particles. For particles that did overlap, we also extracted the center-

716 to-center distance of those overlapping particles. For all data, analysis of

717 variation (ANOVA) for multiple comparisons was performed with Kaleidagraph

718 (Synergy) using the Tukey HSD post hoc tests. The alpha for statistical

719 significance was set to 0.05. For pairwise comparisons, a student-T test was used 720 using Kaleidagraph (Synergy).

\section{Author contributions}

M.C. and M.B. designed the research. M.C., S.W., J.E.O. and S.E.R performed the research. M.C., S.W. and M.B. wrote the manuscript.

\section{Acknowledgements}

We thank members of the Bezanilla lab for careful reading of the manuscript. M.C. received support from the Plant Biology Graduate Program at the University of Massachusetts, Amherst. This work was supported by Dartmouth College and grants from the National Science Foundation (MCB1330171 and MCB-1715785 to M.B.).

\section{References}

735 Aridor, M. (2018). COPII gets in shape: Lessons derived from morphological aspects of early secretion. Traffic 19: 823-839.

Aridor, M., Fish, K.N., Bannykh, S., Weissman, J., Roberts, T.H., LippincottSchwartz, J., and Balch, W.E. (2001). The Sar1 Gtpase Coordinates Biosynthetic Cargo Selection with Endoplasmic Reticulum Export Site Assembly. Journal of Cell Biology 152: 213-230.

Aridor, M., Weissman, J., Bannykh, S., Nuoffer, C., and Balch, W.E. (1998). Cargo Selection by the COPII Budding Machinery during Export from the ER. Journal of Cell Biology 141: 61-70. 
Augustine, R.C., Pattavina, K.A., Tüzel, E., Vidali, L., and Bezanilla, M. (2011). Actin interacting protein 1 and actin depolymerizing factor drive rapid actin dynamics in Physcomitrella patens. Plant Cell 23: 3696-3710.

Barlow, L.D. and Dacks, J.B. (2018). Seeing the endomembrane system for the trees: Evolutionary analysis highlights the importance of plants as models for eukaryotic membrane-trafficking. Seminars in Cell \& Developmental Biology 80: 142-152.

Barlowe, C. (2003). Signals for COPII-dependent export from the ER: what's the ticket out? Trends in Cell Biology 13: 295-300.

Barlowe, C. and Helenius, A. (2016). Cargo Capture and Bulk Flow in the Early Secretory Pathway. Annu. Rev. Cell Dev. Biol. 32: 197-222.

Barlowe, C. and Schekman, R. (1993). SEC12 encodes a guanine-nucleotideexchange factor essential for transport vesicle budding from the ER. Nature 365: 347-349.

Bascom, C.S., Wu, S.-Z., Nelson, K., Oakey, J., and Bezanilla, M. (2016). Long-Term Growth of Moss in Microfluidic Devices Enables Subcellular Studies in Development. Plant Physiology 172: 28-37.

Belden, W.J. (2001). Role of Erv29p in Collecting Soluble Secretory Proteins into ER-Derived Transport Vesicles. Science 294: 1528-1531.

Bezanilla, M., Perroud, P.-F., Pan, A., Klueh, P., and Quatrano, R.S. (2005). An RNAi system in Physcomitrella patens with an internal marker for silencing allows for rapid identification of loss of function phenotypes. Plant Biol (Stuttg) 7: 251-257.

Bi, X., Corpina, R.A., and Goldberg, J. (2002). Structure of the Sec23/24-Sar1 prebudding complex of the COPII vesicle coat. Nature 419: 271-277.

Brandizzi, F. (2018). Transport from the endoplasmic reticulum to the Golgi in plants: Where are we now? Seminars in Cell \& Developmental Biology 80: 94-105.

Brandizzi, F. and Barlowe, C. (2013). Organization of the ER-Golgi interface for membrane traffic control. Nat Rev Mol Cell Biol 14: 382-392.

Cheng, F-y., Zamski, E., Guo W-w., Mason Pharr, D., and Williamson, J.D. (2009). Salicylic acid stimulates secretion of the normally symplastic enzyme mannitol dehydrogenase: a possible defense against mannitol-secreting fungal pathogens. Planta 230: 1093-1103.

Cheng, X., and Bezanilla M. (2021). SABRE populates ER domains essential for cell plate maturation and cell expansion influencing cell and tissue patterning. eLife 10: e65166. 
Cho, Y. and Kanehara, K. (2017). Endoplasmic Reticulum Stress Response in Arabidopsis Roots. Front. Plant Sci. 8.

Chung, K.P., Zeng, Y., and Jiang, L. (2016). COPII Paralogs in Plants: Functional Redundancy or Diversity? Trends in Plant Science 21: 758-769.

De Craene, J.-O., Courte, F., Rinaldi, B., Fitterer, C., Herranz, M.C., SchmittKeichinger, C., Ritzenthaler, C., and Friant, S. (2014). Study of the Plant COPII Vesicle Coat Subunits by Functional Complementation of Yeast Saccharomyces cerevisiae Mutants. PLoS ONE 9: e90072.

Ding, Y., Wang, J., Wang, J., Stierhof, Y-D., Robinson, D.G., and Jiang L. (2012). Unconventional protein secretion. Trends in Plant Science 17: 606-615.

Faso, C., Chen, Y.-N., Tamura, K., Held, M., Zemelis, S., Marti, L., Saravanan, R., Hummel, E., Kung, L., Miller, E., Hawes, C., and Brandizzi, F. (2009). A Missense Mutation in the Arabidopsis COPII Coat Protein Sec24A Induces the Formation of Clusters of the Endoplasmic Reticulum and Golgi Apparatus. The Plant Cell 21: 3655-3671.

Fath, S., Mancias, J.D., Bi, X., and Goldberg, J. (2007). Structure and Organization of Coat Proteins in the COPII Cage. Cell 129: 1325-1336.

Furt, F., Lemoi, K., Tüzel, E., and Vidali, L. (2012). Quantitative analysis of organelle distribution and dynamics in Physcomitrella patens protonemal cells. BMC Plant Biol. 12: 70.

Galotto, G., Bibeau, J.P., and Vidali, L. (2019). Automated Image Acquisition and Morphological Analysis of Cell Growth Mutants in Physcomitrella patens. In Plant Cell Morphogenesis, F. Cvrčková and V. Žárský, eds, Methods in Molecular Biology. (Springer New York: New York, NY), pp. 307-322.

Gilles, J.-F., Dos Santos, M., Boudier, T., Bolte, S., and Heck, N. (2017). DiAna, an Image tool for object-based 3D co-localization and distance analysis. Methods 115: 55-64.

Giraudo, C.G. and Maccioni, H.J.F. (2003). Endoplasmic Reticulum Export of Glycosyltransferases Depends on Interaction of a Cytoplasmic Dibasic Motif with Sar1. MBoC 14: 3753-3766.

van Gisbergen, P.A.C., Wu, S.-Z., Chang, M., Pattavina, K.A., Bartlett, M.E., and Bezanilla, M. (2018). An ancient Sec10-formin fusion provides insights into actin-mediated regulation of exocytosis. J. Cell Biol. 217: 945-957.

Haeussler, M., Schönig, K., Eckert, H., Eschstruth, A., Mianné, J., Renaud, J.-B., Schneider-Maunoury, S., Shkumatava, A., Teboul, L., Kent, J., Joly, J.-S., and Concordet, J.-P. (2016). Evaluation of off-target and on-target scoring 

algorithms and integration into the guide RNA selection tool CRISPOR. Genome Biol. 17: 148.

Hanna, M.G., Peotter, J.L., Frankel, E.B., and Audhya, A. (2018). Membrane Transport at an Organelle Interface in the Early Secretory Pathway: Take Your Coat Off and Stay a While: Evolution of the metazoan early secretory pathway. BioEssays 40: 1800004.

Hanton, S.L., Matheson, L.A., Chatre, L., and Brandizzi, F. (2009). Dynamic organization of COPII coat proteins at endoplasmic reticulum export sites in plant cells. The Plant Journal 57: 963-974.

Heinze, L., Freimuth, N., Rößling, A.-K., Hahnke, R., Riebschläger, S., Fröhlich, A., Sampathkumar, A., McFarlane, H.E., and Sauer, M. (2020). EPSIN1 and MTV1 define functionally overlapping but molecularly distinct trans-Golgi network subdomains in Arabidopsis. PNAS 117: 25880-25889.

Hino, T., Tanaka, Y., Kawamukai, M., Nishimura, K., Mano, S., and Nakagawa, T. (2011). Two Sec13p Homologs, AtSec13A and AtSec13B, Redundantly Contribute to the Formation of COPII Transport Vesicles in Arabidopsis thaliana. Bioscience, Biotechnology, and Biochemistry 75: 1848-1852.

Hiwatashi, Y., Sato, Y., and Doonan, J.H. (2014). Kinesins Have a Dual Function in Organizing Microtubules during Both Tip Growth and Cytokinesis in Physcomitrella patens. The Plant Cell 26: 1256-1266.

Jensen, D. and Schekman, R. (2011). COPII-mediated vesicle formation at a glance. Journal of Cell Science 124: 1-4.

Khoriaty, R. et al. (2018). Functions of the COPII gene paralogs SEC23A and SEC23B are interchangeable in vivo. Proc Natl Acad Sci USA 115: E7748E7757.

Lee, M.H., Lee, S.H., Kim, H., Jin, J.B., Kim, D.H., and Hwang, I. A WD40 Repeat Protein, Arabidopsis Sec13 Homolog 1, May Play a Role in Vacuolar Trafficking by Controlling the Membrane Association of AtDRP2A.: 10.

Mallett, D.R., Chang, M., Cheng, X., and Bezanilla, M. (2019). Efficient and modular CRISPR-Cas9 vector system for Physcomitrella patens. Plant Direct 3: e00168-15.

Mancias, J.D. and Goldberg, J. (2007). The Transport Signal on Sec22 for Packaging into COPII-Coated Vesicles Is a Conformational Epitope. Molecular Cell 26: 403-414.

Maruyama, D., Sugiyama, T., Endo, T., and Nishikawa, S. (2014). Multiple BiP Genes of Arabidopsis thaliana are Required for Male Gametogenesis and Pollen Competitiveness. Plant and Cell Physiology 55: 801-810. 
Mossessova, E., Bickford, L.C., and Goldberg, J. (2003). SNARE selectivity of the COPII coat. Cell 114: 483-495.

Nakano, A. and Muramatsu, M. (1989). A Novel GTP-binding Protein, Sarlp, Is Involved in Transport from the Endoplasmic Reticulum to the Golgi Apparatus. The Journal of Cell Biology 109: 2677-2691.

Nakano, R.T., Matsushima, R., Ueda, H., Tamura, K., Shimada, T., Li, L., Hayashi, Y., Kondo, M., Nishimura, M., and Hara-Nishimura, I. (2009). GNOMLIKE1/ERM01 and SEC24a/ERMO2 Are Required for Maintenance of Endoplasmic Reticulum Morphology in Arabidopsis thaliana. The Plant Cell 21: 3672-3685.

Nebenfuhr, A. (2002). Brefeldin A: Deciphering an Enigmatic Inhibitor of Secretion. Plant physiology 130: 1102-1108.

Nelson, B.K., Cai, X., and Nebenführ, A. (2007). A multicolored set of in vivo organelle markers for co-localization studies in Arabidopsis and other plants. Plant J. 51: 1126-1136.

Niu, T.-K., Pfeifer, A.C., Lippincott-Schwartz, J., and Jackson, C.L. (2005). Dynamics of GBF1, a Brefeldin A-Sensitive Arf1 Exchange Factor at the GolgiøV. Molecular Biology of the Cell 16: 1213-1222.

Noh, S.-J., Kwon, C.S., Oh, D.-H., Moon, J.S., and Chung, W.-I. (2003). Expression of an evolutionarily distinct novel BiP gene during the unfolded protein response in Arabidopsis thaliana. Gene 311: 81-91.

Novick, P., Field, C., and Schekman, R. (1980). Identification of 23 complementation groups required for post-translational events in the yeast secretory pathway. Cell 21: 205-215.

Nufer, O. (2003). ER export of ERGIC-53 is controlled by cooperation of targeting determinants in all three of its domains. Journal of Cell Science 116: 4429-4440.

Ortiz-Ramírez, C., Michard, E., Simon, A.A., Damineli, D.S.C., Hernandez-Coronado, M., Becker, J.D., and Feijó, J.A. (2017). GLUTAMATE RECEPTOR-LIKE channels are essential for chemotaxis and reproduction in mosses. Nature 549: 91-95.

Qu, X., Zhang, R., Zhang, M., Diao, M., Xue, Y., and Huang, S. (2017). Organizational innovation of apical actin filaments drives rapid pollen tube growth and turning. Mol Plant 10: 930-947.

Rensing, S.A., Goffinet, B., Meyberg, R., Wu, S.-Z., and Bezanilla, M. (2020). The Moss Physcomitrium ( Physcomitrella) patens : A Model Organism for Non-Seed Plants. Plant Cell 32: 1361-1376. 
Robinson, D.G., Brandizzi, F., Hawes, C., and Nakano, A. (2015). Vesicles versus Tubes: Is Endoplasmic Reticulum-Golgi Transport in Plants Fundamentally Different from Other Eukaryotes? Plant Physiology 168: 393-406.

Salama, N.R., Yeung, T., and Schekman, R.W. The Secl3p complex and reconstitution of vesicle budding from the ER with purified cytosolic proteins. The EMBO Journal 12: 4073-4082.

Scheuring, D., Schöller, M., Kleine-Vehn, J., and Löfke, C. (2015). Vacuolar staining methods in plant cells. Methods Mol Biol 1242: 83-92.

Schlacht, A. and Dacks, J.B. (2015). Unexpected Ancient Paralogs and an Evolutionary Model for the COPII Coat Complex. Genome Biology and Evolution 7: 1098-1109.

Srivastava, R., Deng, Y., Shah, S., Rao, A.G., and Howell, S.H. (2013). BINDING PROTEIN Is a Master Regulator of the Endoplasmic Reticulum Stress Sensor/Transducer bZIP28 in Arabidopsis. Plant Cell 25: 1416-1429.

Stagg, S.M., Gürkan, C., Fowler, D.M., LaPointe, P., Foss, T.R., Potter, C.S., Carragher, B., and Balch, W.E. (2006). Structure of the Sec13/31 COPII coat cage. Nature 439: 234-238.

Stagg, S.M., LaPointe, P., Razvi, A., Gürkan, C., Potter, C.S., Carragher, B., and Balch, W.E. (2008). Structural Basis for Cargo Regulation of COPII Coat Assembly. Cell 134: 474-484.

Takagi, J., Renna, L., Takahashi, H., Koumoto, Y., Tamura, K., Stefano, G., Fukao, Y., Kondo, M., Nishimura, M., Shimada, T., Brandizzi, F., and Hara-Nishimura, I. (2013). MAIG05 Functions in Protein Export from Golgi-Associated Endoplasmic Reticulum Exit Sites in Arabidopsis. The Plant Cell 25: 46584675.

Tanaka, Y., Nishimura, K., Kawamukai, M., Oshima, A., and Nakagawa, T. (2013). Redundant function of two Arabidopsis COPII components, AtSec24B and AtSec24C, is essential for male and female gametogenesis. Planta 238: 561-575.

Vidali, L., Augustine, R.C., Kleinman, K.P., and Bezanilla, M. (2007). Profilin is essential for tip growth in the moss Physcomitrella patens. Plant Cell 19: 3705-3722.

Vidali, L., van Gisbergen, P.A.C., Guérin, C., Franco, P., Li, M., Burkart, G.M., Augustine, R.C., Blanchoin, L., and Bezanilla, M. (2009). Rapid forminmediated actin-filament elongation is essential for polarized plant cell growth. Proc. Natl. Acad. Sci. U.S.A. 106: 13341-13346. 
989

990

991

992

993

994

995

996

997

998

999

1000

1001

1002

1003

1004

1005

1006

1007

1008

1009

1010

1011

1012

1013

1014

1015

1016

1017

1018

1019

1020

1021

1022

1023

1024

1025

1026

1027

1028

1029

1030

1031

1032

Viotti, C. et al. (2013). The Endoplasmic Reticulum Is the Main Membrane Source for Biogenesis of the Lytic Vacuole in Arabidopsis. Plant Cell 25: 34343449.

Wang, X., Chung, K. P., Lin, W., and Jiang L. (2017). Protein secretion in plants: conventional and unconventional pathways and new techniques. Journal of Experimental Botany 69: 21-37.

Whittle, J.R.R. and Schwartz, T.U. (2010). Structure of the Sec13-Sec16 edge element, a template for assembly of the COPII vesicle coat. The Journal of Cell Biology 190: 347-361.

Wu, S.-Z. and Bezanilla, M. (2014). Myosin VIII associates with microtubule ends and together with actin plays a role in guiding plant cell division. eLife Sciences 3: e03498.

Wu, S.-Z., Yamada, M., Mallett, D.R., and Bezanilla, M. (2018). Cytoskeletal discoveries in the plant lineage using the moss Physcomitrella patens. Biophys Rev 10: 1683-1693.

Zeng, Y., Chung, K.P., Li, B., Lai, C.M., Lam, S.K., Wang, X., Cui, Y., Gao, C., Luo, M., Wong, K.-B., Schekman, R., and Jiang, L. (2015). Unique COPII component AtSar1a/AtSec23a pair is required for the distinct function of protein ER export in Arabidopsis thaliana. Proc Natl Acad Sci USA 112: 14360-14365.

Zeng, Y., Li, B., Ji, C., Feng, L., Niu, F., Deng, C., Chen, S., Lin, Y., Cheung, K.C.P., Shen, J., Wong, K.-B., and Jiang, L. (2021). A unique AtSar1D-AtRabD2a nexus modulates autophagosome biogenesis in Arabidopsis thaliana. Proc Natl Acad Sci U S A 118.

Zhang, H., Zhang, L., Gao, B., Fan, H., Jin, J., Botella, M.A., Jiang, L., and Lin, J. (2011). Golgi apparatus-localized synaptotagmin 2 is required for unconventional secretion in Arabidopsis. PLoS ONE 6: e26477.

\section{Figure Legends}

Figure 1. Sec23 and Sec24 are required for polarized growth. Gene models of the P. patens Sec23 (A) and Sec24 (B) genes are shown with exons indicated by boxes and introns by thin black lines. Coding and untranslated regions are denoted by thick and thin boxes, respectively. The lines underneath the gene models represent sequence regions targeted by RNAi constructs. Scale bar is $500 \mathrm{bp}$. Graphs depict the relative expression of Sec23 and Sec24 genes normalized to UBIQUITIN10 in 8-day-old wild-type moss plants regenerated from protoplasts. $\mathrm{N}=3$, Error bars are s.e.m. (C, E) Representative chlorophyll autofluorescence images of 7-day-old plants regenerated from protoplasts 
1033 expressing the indicated RNAi constructs. Scale bar, $100 \mu \mathrm{m}$. (D, F)

1034 Quantification of plant area is based on the area of the chlorophyll

1035 autofluorescence and is presented normalized to the area of the control for each

1036 experiment. Means are indicated by horizontal lines. Letters indicate groups with

1037 significantly different means as determined by a one-way ANOVA with a Tukey

1038 post hoc test $(\alpha=0.05)$.

1039

1040 Figure 2. Sec23D and Sec23E are functionally redundant. (A) Sec23D and

1041 Sec23E gene models are shown with exons indicated by boxes and introns by

1042 thin black lines. Coding and untranslated regions are denoted by thick and thin

1043 boxes, respectively. The lines underneath the gene models represent sequence

1044 regions that were targeted by Sec23D or Sec23E UTR RNAi constructs. Scale bar

1045 is 500bp. (B) Representative chlorophyll autofluorescence images of 7-day-old

1046 plants regenerated from protoplasts expressing the indicated constructs. Scale

1047 bar, $100 \mu \mathrm{m}$. (C) Quantification of plant area was normalized to the control for

1048 each experiment. Letters indicate groups with significantly different means as

1049 determined by a one-way ANOVA with a Tukey post hoc test $(\alpha=0.05)$.

1050

1051 Figure 3. CRISPR-Cas9-mediated deletion of Sec23 isoforms demonstrates

1052 that Sec23D is critical for protonemal growth. (A) Representative chlorophyll

1053 autofluorescence images of 7-day old plants with the indicated genotype

1054 regenerated from protoplasts. Scale bar, $100 \mu \mathrm{m}$. (B) Quantification of plant area

1055 normalized to the wild type control. (C) Representative 3-week old moss plants

1056 with the indicated genotype. Scale bar, 1mm. (D) Quantification of the plant area

1057 of 3-week old moss plants normalized to that of the wild type control. (E)

1058 Number of gametophores in 3-week old moss plants. Letters in (B, D-E) indicate

1059 groups with significantly different means as determined by a one-way ANOVA

1060 with a Tukey post hoc test $(\alpha=0.05)$. (F) Relative expression of Sec23D and $E$

1061 genes normalized to UBIQUITIN10 in 8-day-old wild type and $\Delta s e c 23 a b c f g-1$, and

$1062 \Delta s e c 23 a b c f g-2$ moss plants regenerated from protoplasts. $\mathrm{N}=3$, Error bars are

1063 s.e.m. No significant difference was determined by a one-way ANOVA with a

1064 Tukey post hoc test $(\alpha=0.05)$. 
1066

1067

1068

1069

1070

1071

1072

1073

1074

1075

1076

1077

1078

1079

1080

1081

1082

1083

1084

1085

1086

1087

1088

1089

1090

1091

1092

1093

1094

1095

1096

1097

1098

Figure 4. Loss of Sec23D causes ER morphology defects and ER stress. (A)

Laser scanning confocal images of the ER labeled with GFP-KDEL in the indicated genotype. Colored boxes highlight large and small ER aggregates found in the $\Delta$ sec23d-B11 mutant. Scale bars, $5 \mu \mathrm{m}$. (B) Spinning disc confocal super resolution imaging of the ER labeled with GFP-KDEL in a $\Delta s e c 23 d-B 11$ cell. Scale bar, $5 \mu \mathrm{m}$. Box outlined with yellow dotted line is enlarged to the right and the box outlined with a white dotted line is enlarged below with optical sections spaced every $0.5 \mu \mathrm{m}$ shown for this region of the cell. Scale bars in boxed regions, $2 \mu \mathrm{m}$. Also see Movie S1. (C, D) Relative expression of PpBip1 in 8-day old moss plants regenerated from protoplasts. N=3, Error bars are s.e.m. Asterisks denote t-probability is $<0.01$. n.s. denotes no significant difference. In (B) BFA-treated plants were normalized to DMSO-treated plants and in (C) null mutants were normalized to wild type plants.

\section{Figure 5. Deletion of $\operatorname{Sec} 23 \mathrm{D}$ inhibits Golgi trafficking and secretion to the} plasma membrane. (A) Representative maximum projections of confocal images of the indicated genotypes expressing a YFP-MAN marker. Scale bar, 10 $\mu \mathrm{m}$. (B) Quantification of the fluorescence intensity ratio within the Golgi to the background. (C) Representative medial sections of confocal images of the indicated genotypes expressing a plasma membrane marker (FSnap-mCherry). Scale bar, $10 \mu \mathrm{m}$. (D) Quantification of the fluorescence intensity ratio of the plasma membrane to the intensity within the cell. Letters in $(B, D)$ indicate groups with significantly different means as determined by a one-way ANOVA with a Tukey post hoc test $(\alpha=0.05)$.

\section{Figure 6. Sec23 isoforms localize to ER proximal structures with distinct} sizes. (A) Representative confocal Z-sections of lines where the indicated Sec23 gene was endogenously tagged with 3XmRuby in a line that expresses GFP-KDEL labeling the ER lumen (ER-GFP). Scale bars, $5 \mu \mathrm{m}$. Boxed region in the mRuby channel is shown to the right of each image. (B) Pearson's correlation coefficient measured between the Sec23 labeled lines and ER-GFP. 0, original ER image; V, vertically flipped ER image; $H$, horizontally flipped ER image. Asterisks denote $\mathrm{p}<0.001$ as determined by a one-way ANOVA with a Tukey post hoc test $(\alpha=0.05)$ 
1099 (C) Representative confocal Z-sections of Sec23 genes endogenously tagged with

1100 3XmRuby acquired with the optimal resolution of the confocal imaging system.

1101 Scale bars, $5 \mu \mathrm{m}$. (D) Quantification of average dot size in the Sec23 images as

1102 determined by the diAna tool for Fiji (Gilles et al., 2017). Asterisks denote

$1103 \mathrm{p}<0.001$ as determined by a one-way ANOVA with a Tukey post hoc test $(\alpha=0.05)$.

1105 Figure 7. Sec23B and Sec23G form ER exit sites independent of Sec23D.

1106 Representative confocal Z-sections of Sec23D-mRuby lines where Sec23B (A) or

1107 Sec23G (B) were endogenously tagged with mNeon. Scale bar, $5 \mu \mathrm{m}$. Yellow

1108 boxed regions are enlarged to the right of the merged image where Sec23D-

1109 mRuby is false-colored magenta and in (A) Sec23B-mNeon is false-colored green

1110 and in (B) Sec23G-mNeon is false-colored green. (C) Percent of Sec23B-mNeon

1111 or Sec23G-mNeon dots that co-localized with Sec23D-mRuby and (D) center-to-

1112 center distance of co-localized particles as determined using the diAna tool for

1113 Fiji (Gilles et al., 2017). (E) Representative confocal Z-sections of wild type and

$1114 \Delta \sec 23 d-5$ cells with either Sec23B or Sec23G endogenously tagged with

1115 3XmRuby. Scale bar, $5 \mu \mathrm{m}$. (F) Quantification of average dot size as determined

1116 by the diAna tool for Fiji (Gilles et al., 2017). Sec23B and Sec23G dot size in wild

1117 type is the same data presented in Figure 6D. Student t-test was performed for

1118 data in (C, D, and F). The t-probability is indicated for each data pair in each

1119 graph. n.s. denotes no significant difference. 
A

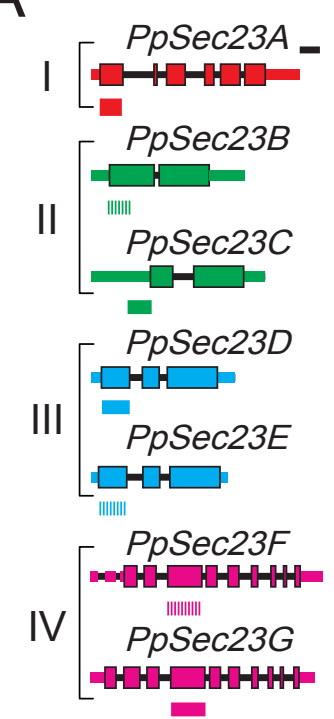

C
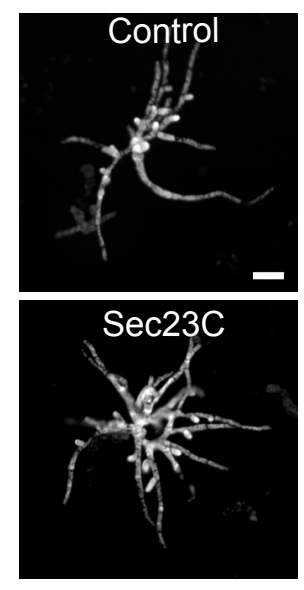

D

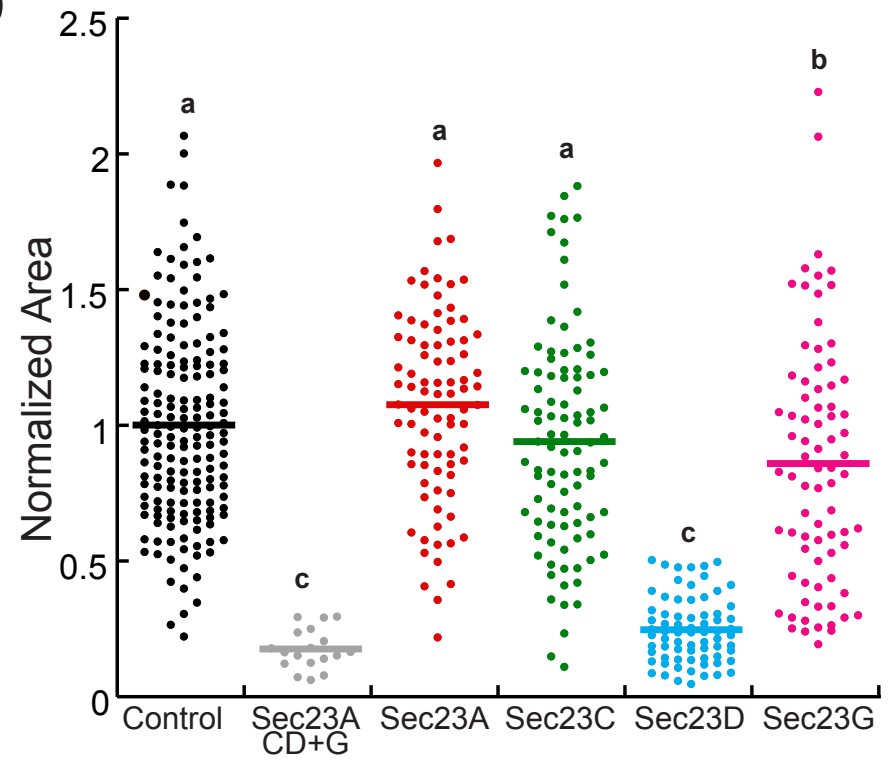

B

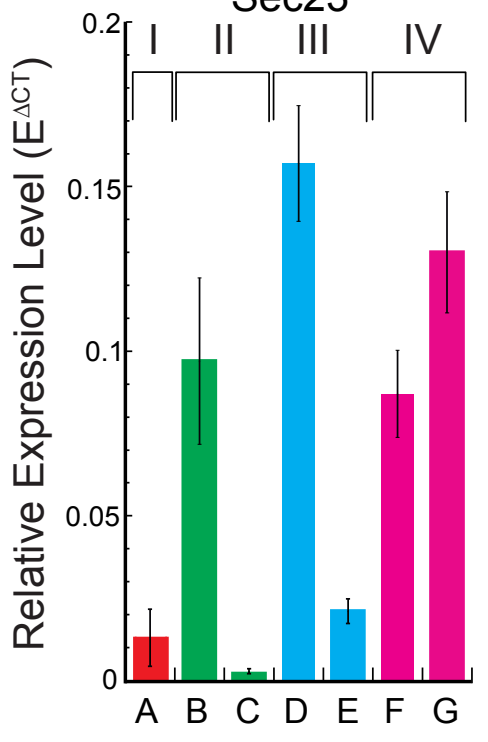

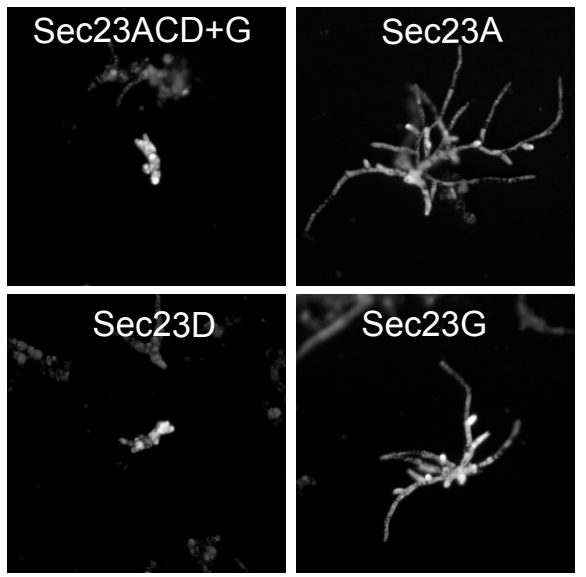

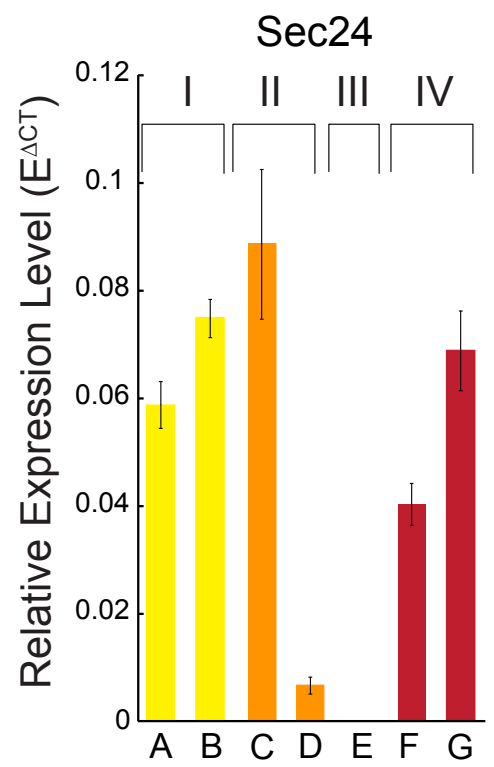

$E$
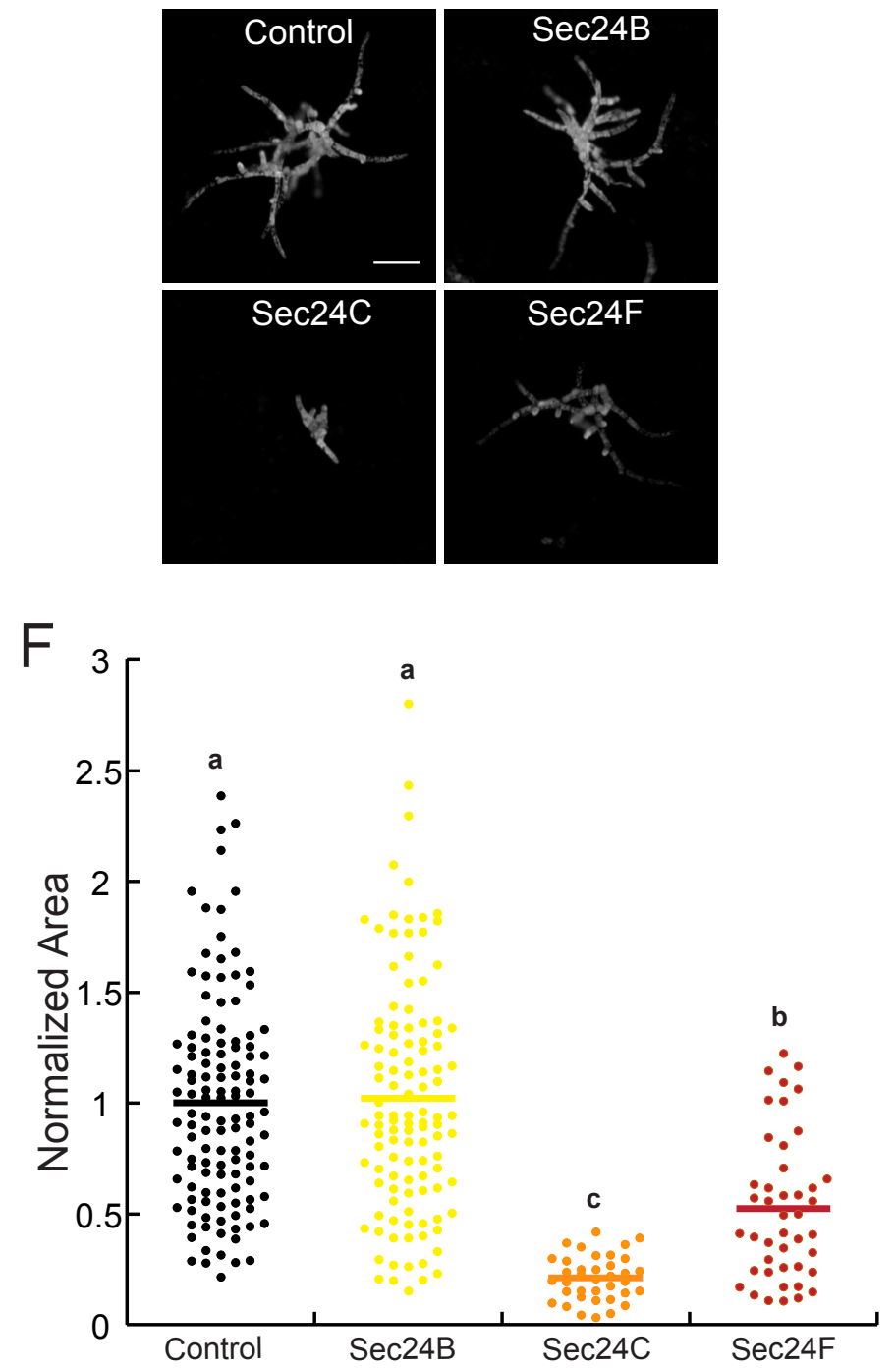
Figure 1. Sec23 and Sec24 are required for polarized growth. Gene models of the P. patens Sec23 (A) and Sec24 (B) genes are shown with exons indicated by boxes and introns by thin black lines. Coding and untranslated regions are denoted by thick and thin boxes, respectively. The lines underneath the gene models represent sequence regions targeted by RNAi constructs. Scale bar is 500bp. Graphs depict the relative expression of Sec23 and Sec24 genes normalized to UBIQUITIN10 in 8-day-old wild-type moss plants regenerated from protoplasts. $\mathrm{N}=3$, Error bars are s.e.m. (C, E) Representative chlorophyll autofluorescence images of 7-day-old plants regenerated from protoplasts expressing the indicated RNAi constructs. Scale bar, $100 \mu \mathrm{m}$. (D, F) Quantification of plant area is based on the area of the chlorophyll autofluorescence and is presented normalized to the area of the control for each experiment. Means are indicated by horizontal lines. Letters indicate groups with significantly different means as determined by a one-way ANOVA with a Tukey post hoc test $(\alpha=0.05)$. 

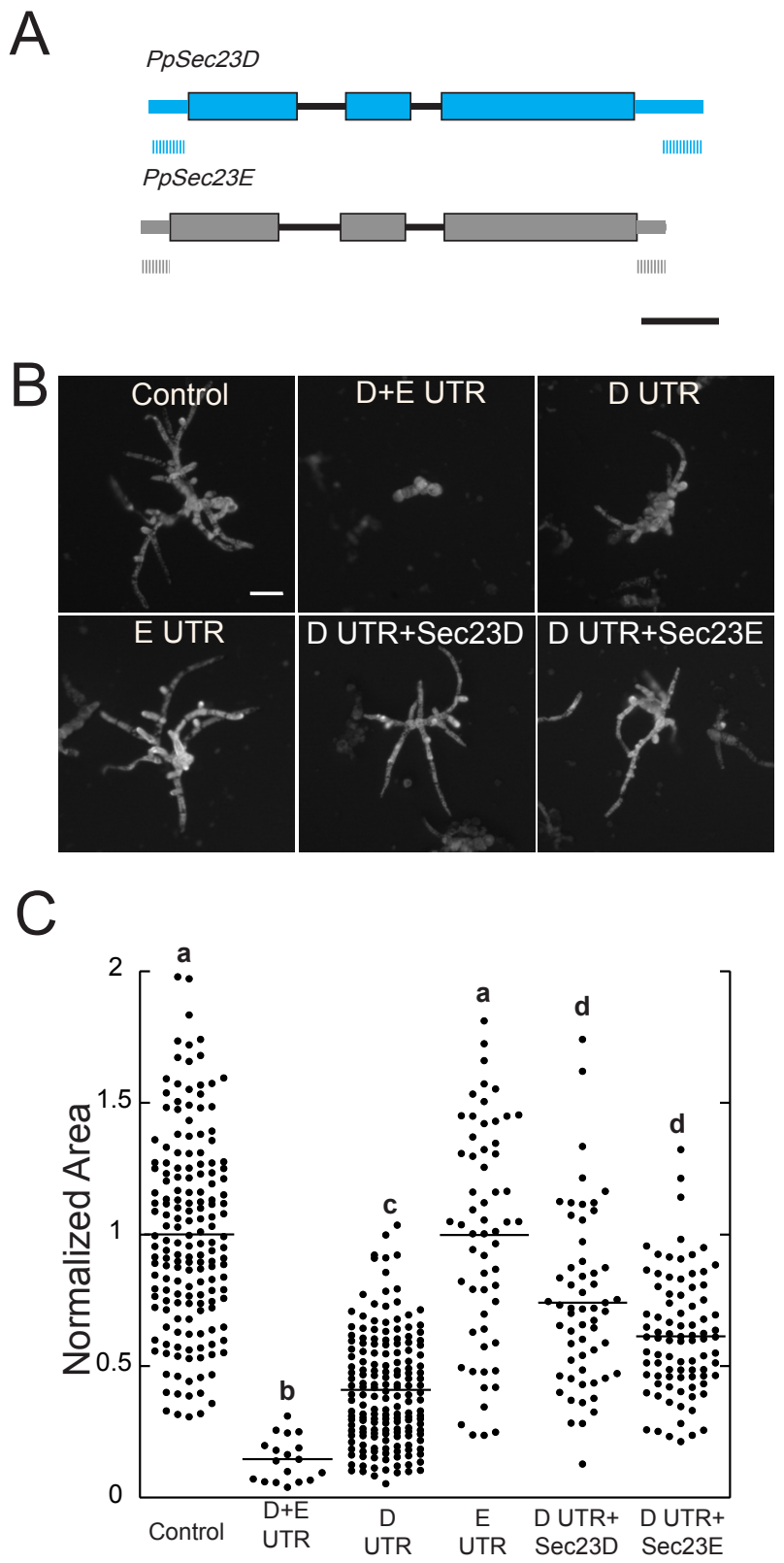

Figure 2. Sec23D and Sec23E are functionally redundant. (A) Sec23D and Sec23E gene models are shown with exons indicated by boxes and introns by thin black lines. Coding and untranslated regions are denoted by thick and thin boxes, respectively. The lines underneath the gene models represent sequence regions that were targeted by Sec23D or Sec23E UTR RNAi constructs. Scale bar is 500bp. (B) Representative chlorophyll autofluorescence images of 7-day-old plants regenerated from protoplasts expressing the indicated constructs. Scale bar, $100 \mu \mathrm{m}$. (C) Quantification of plant area was normalized to the control for each experiment. Letters indicate groups with significantly different means as determined by a one-way ANOVA with a Tukey post hoc test $(\alpha=0.05)$. 

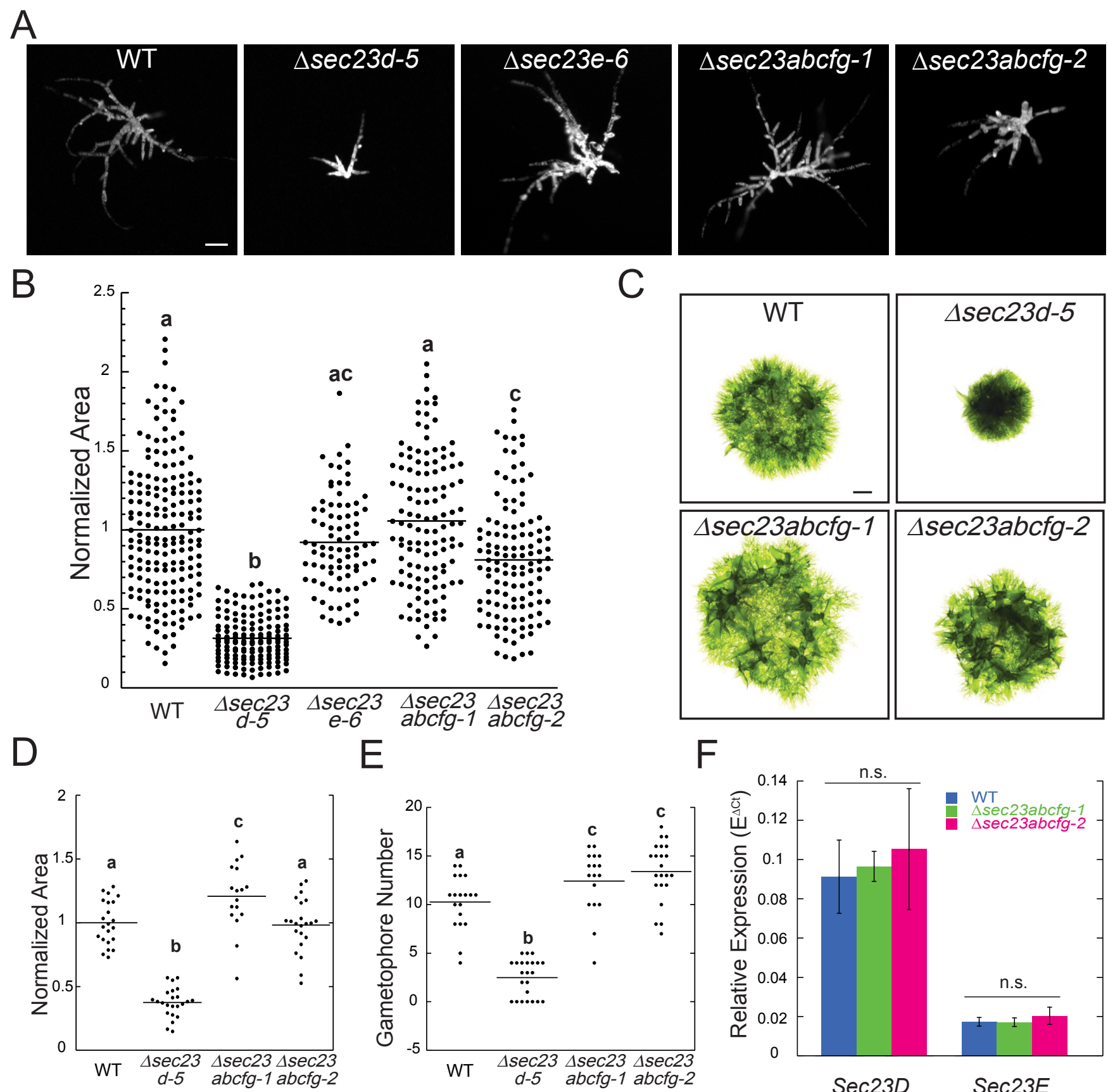

$\mathrm{F}$

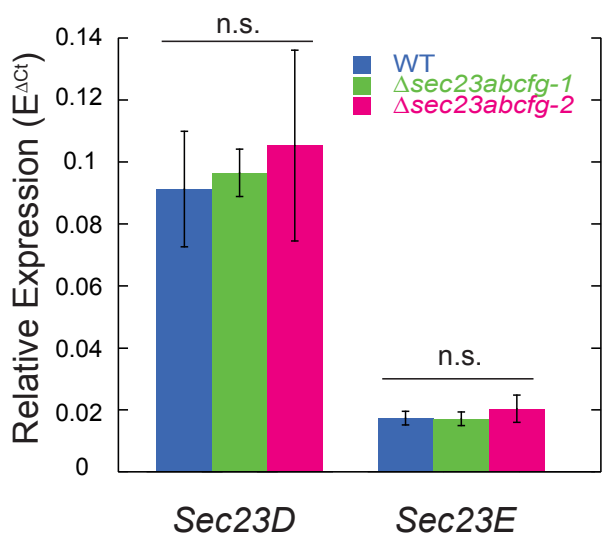

Figure 3. CRISPR-Cas9-mediated deletion of Sec23 isoforms demonstrates that Sec23D is critical for protonemal growth. (A) Representative chlorophyll autofluorescence images of 7-day old plants with the indicated genotype regenerated from protoplasts. Scale bar, $100 \mu \mathrm{m}$. (B) Quantification of plant area normalized to the wild type control. (C) Representative 3-week old moss plants with the indicated genotype. Scale bar, $1 \mathrm{~mm}$. (D) Quantification of the plant area of 3-week old moss plants normalized to that of the wild type control. (E) Number of gametophores in 3-week old moss plants. Letters in (B, D-E) indicate groups with significantly different means as determined by a one-way ANOVA with a Tukey post hoc test $(\alpha=0.05)$. $(F)$ Relative expression of Sec23D and $E$ genes normalized to UBIQUITIN10 in 8-day-old wild type and $\Delta$ sec23abcfg-1, and $\Delta$ sec23abcfg-2 moss plants regenerated from protoplasts. $\mathrm{N}=3$, Error bars are s.e.m. No significant difference was determined by a one-way ANOVA with a Tukey post hoc test $(\alpha=0.05)$. 

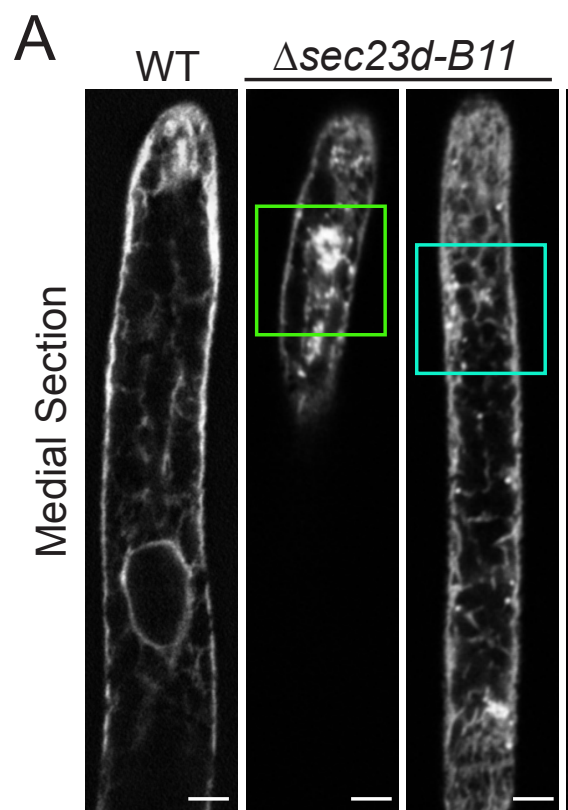

$\triangle \sec 23$ abcfg-1
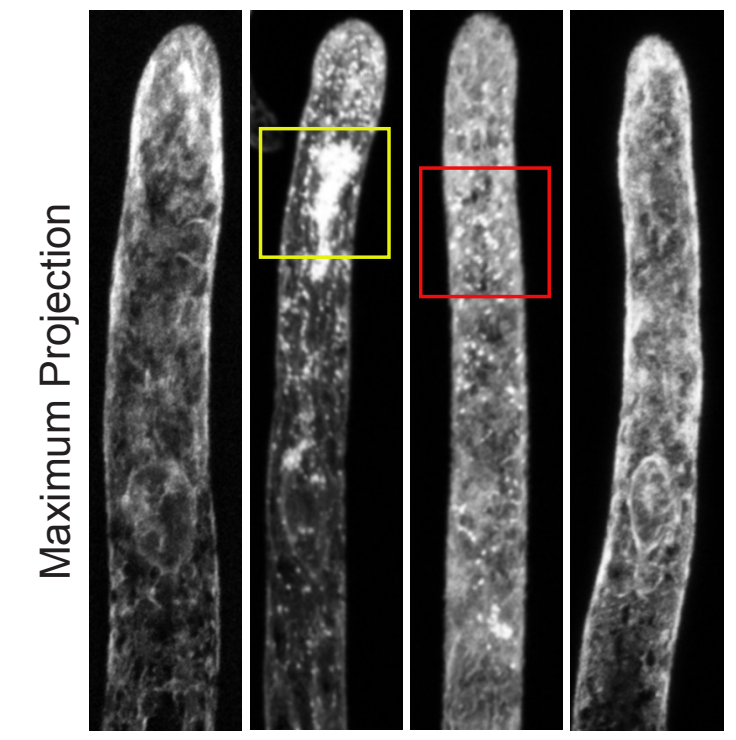

C
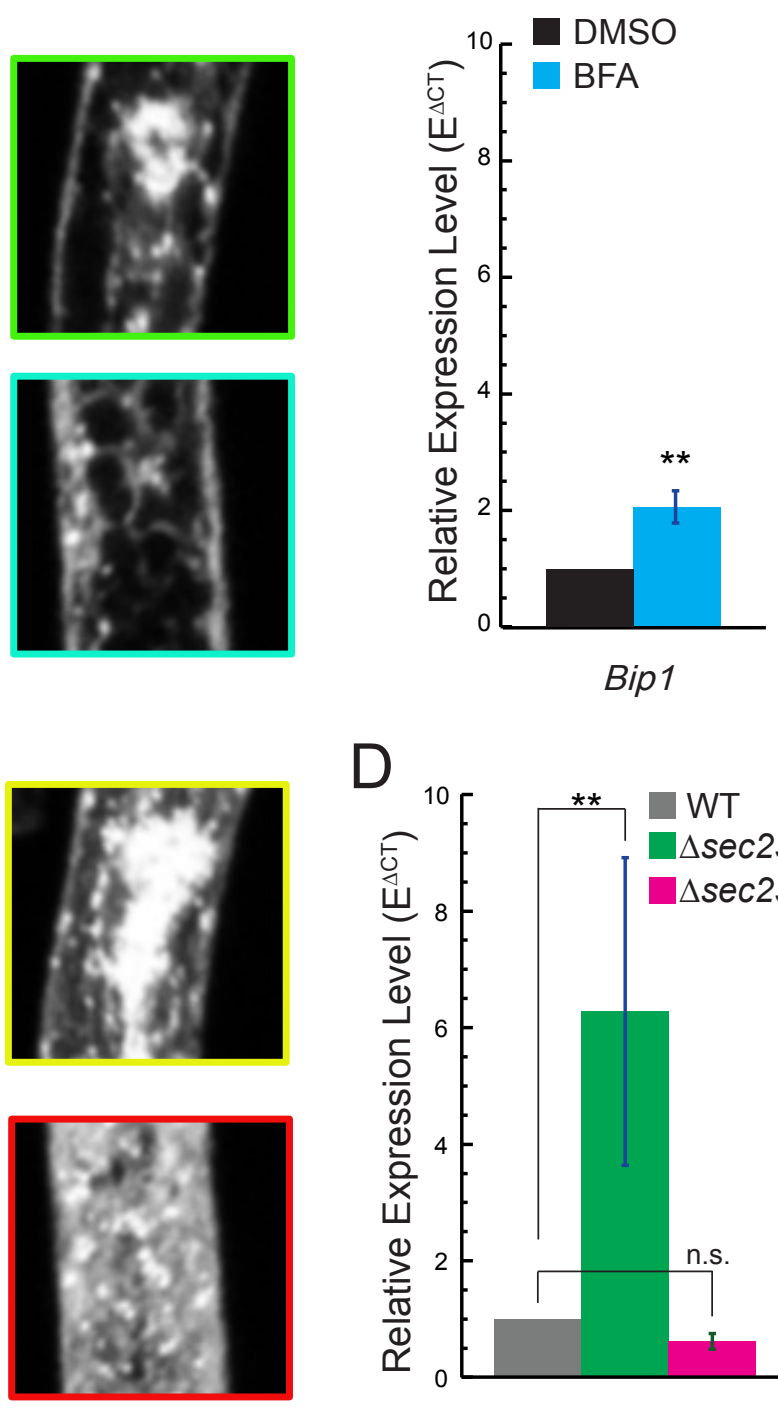

D

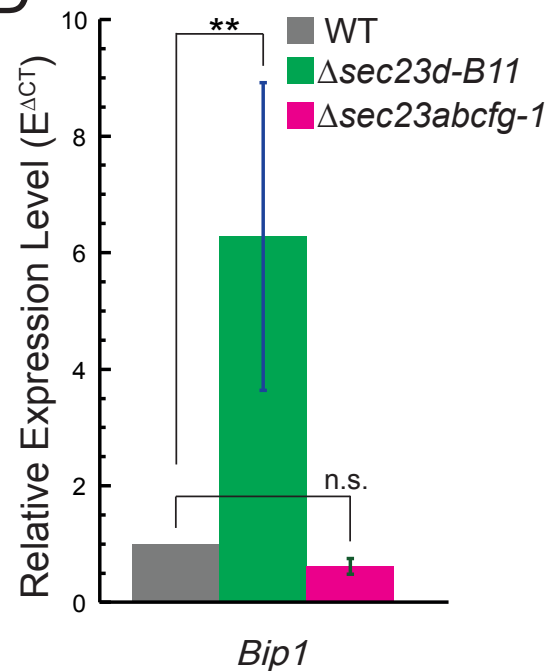

B
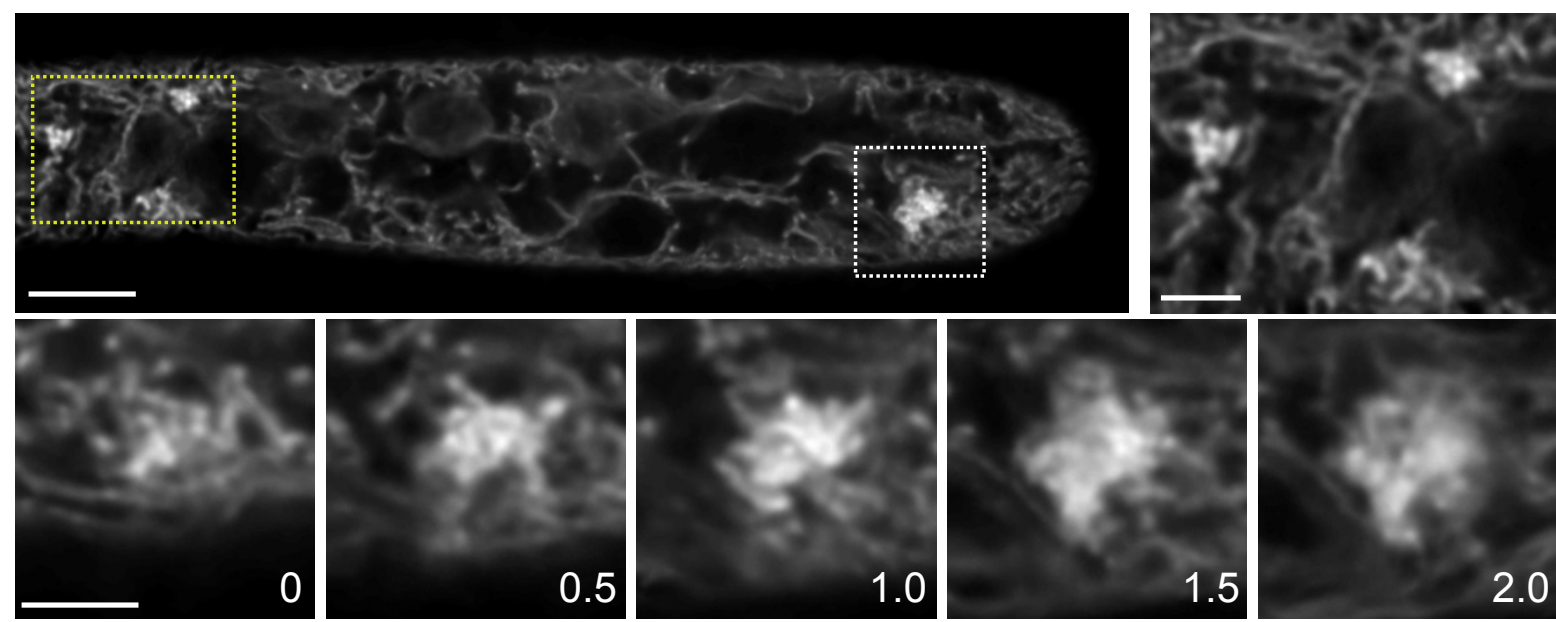

Figure 4. Loss of Sec23D causes ER morphology defects and ER stress. (A) Laser scanning confocal images of the ER labeled with GFP-KDEL in the indicated genotype. Colored boxes highlight large and small ER aggregates found in the $\Delta s e c 23 d-B 11$ mutant. Scale bars, $5 \mu \mathrm{m}$. (B) Spinning disc confocal super resolution imaging of the ER labeled with GFP-KDEL in a $\Delta$ sec23d-B11 cell. Scale bar, 5 $\mu \mathrm{m}$. Box outlined with yellow dotted line is enlarged to the right and the box outlined with a white dotted line is enlarged below with optical sections spaced every $0.5 \mu \mathrm{m}$ shown for this region of the cell. Scale bars in boxed regions, 2 um. Also see Movie S1. (C, D) Relative expression of PpBip1 in 8-day old moss plants regenerated from protoplasts. $\mathrm{N}=3$, Error bars are s.e.m. Asterisks denote t-probability is $<0.01$. n.s. denotes no significant difference. In (B) BFA-treated plants were normalized to DMSO-treated plants and in $(C)$ null mutants were normalized to wild type plants. 
A

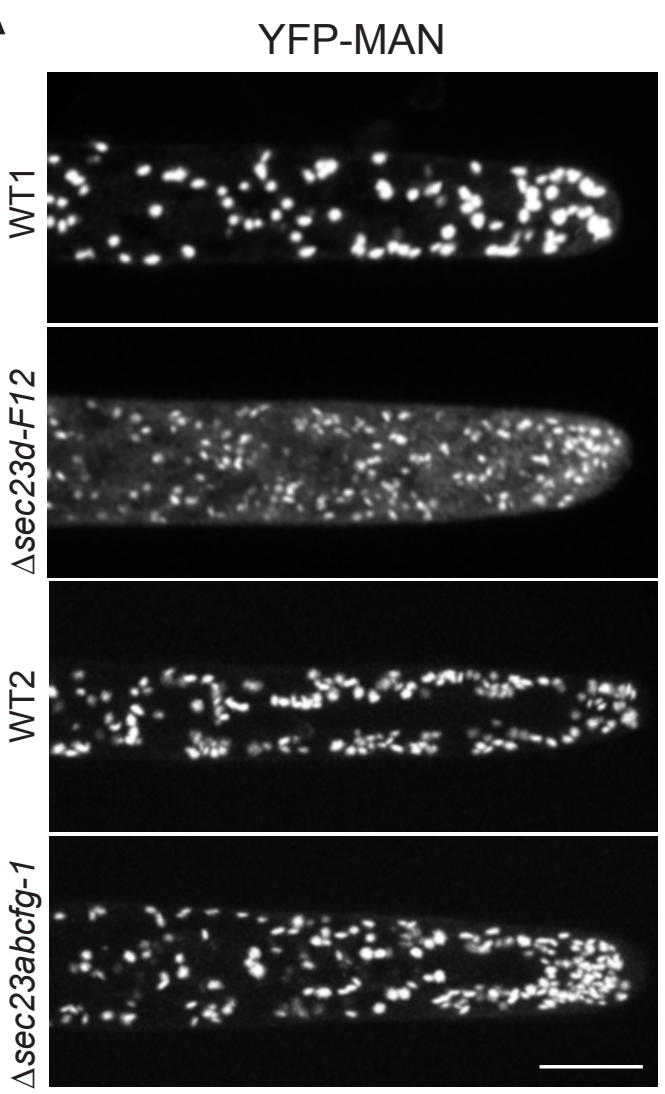

$\mathrm{B}$

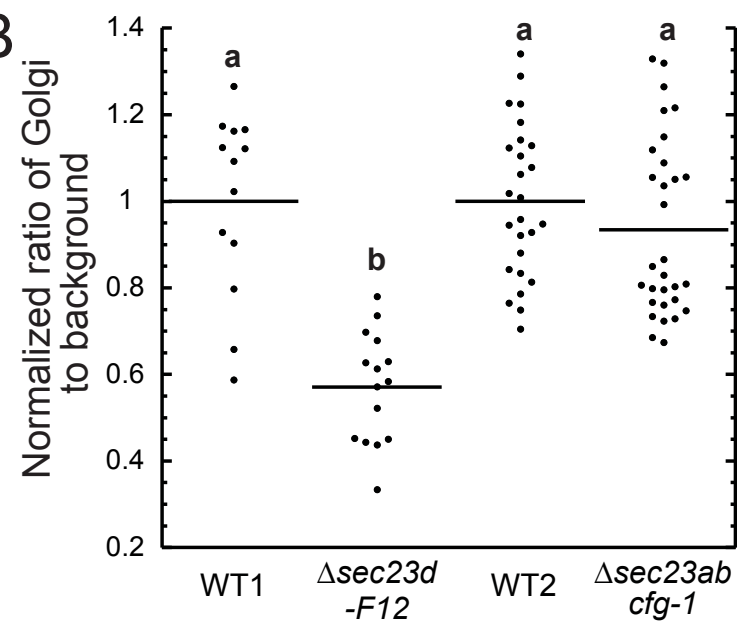

C
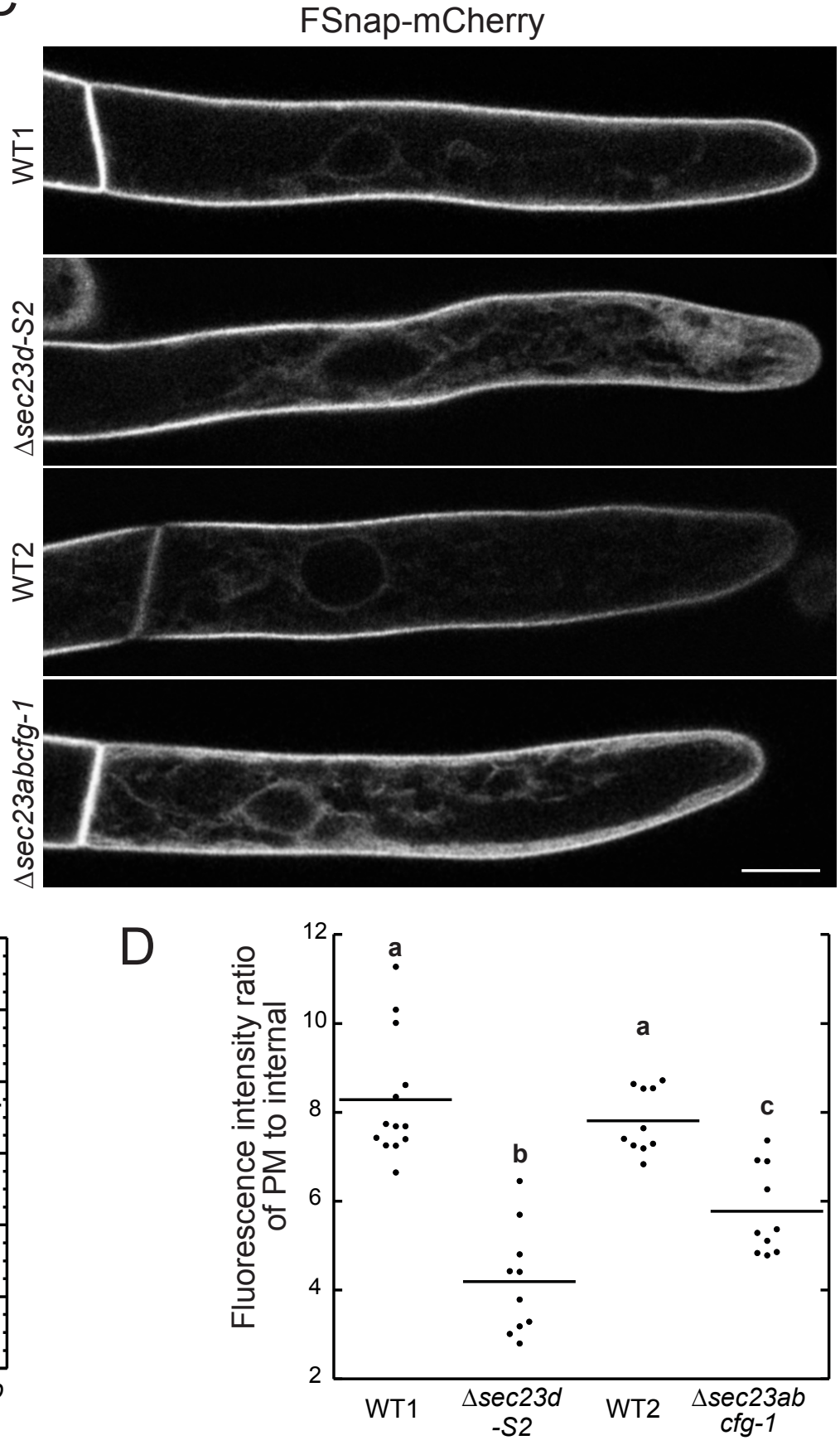

Figure 5. Deletion of Sec23D inhibits Golgi trafficking and secretion to the plasma membrane. (A) Representative maximum projections of confocal images of the indicated genotypes expressing a YFP-MAN marker. Scale bar, $10 \mu \mathrm{m}$. (B) Quantification of the fluorescence intensity ratio within the Golgi to the background. (C) Representative medial sections of confocal images of the indicated genotypes expressing a plasma membrane marker (FSnap-mCherry). Scale bar, $10 \mu \mathrm{m}$. (D) Quantification of the fluorescence intensity ratio of the plasma membrane to the intensity within the cell. Letters in $(B, D)$ indicate groups with significantly different means as determined by a one-way ANOVA with a Tukey post hoc test $(\alpha=0.05)$. 
A
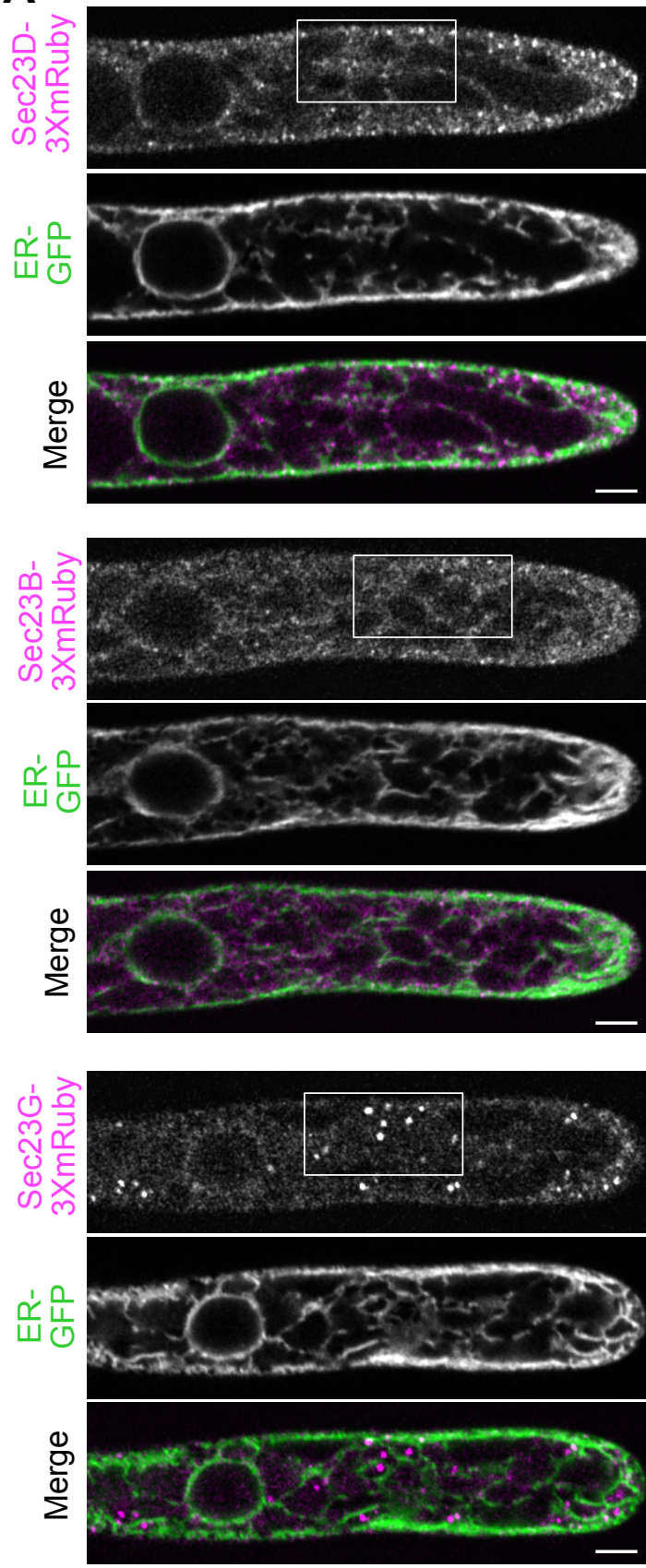

B
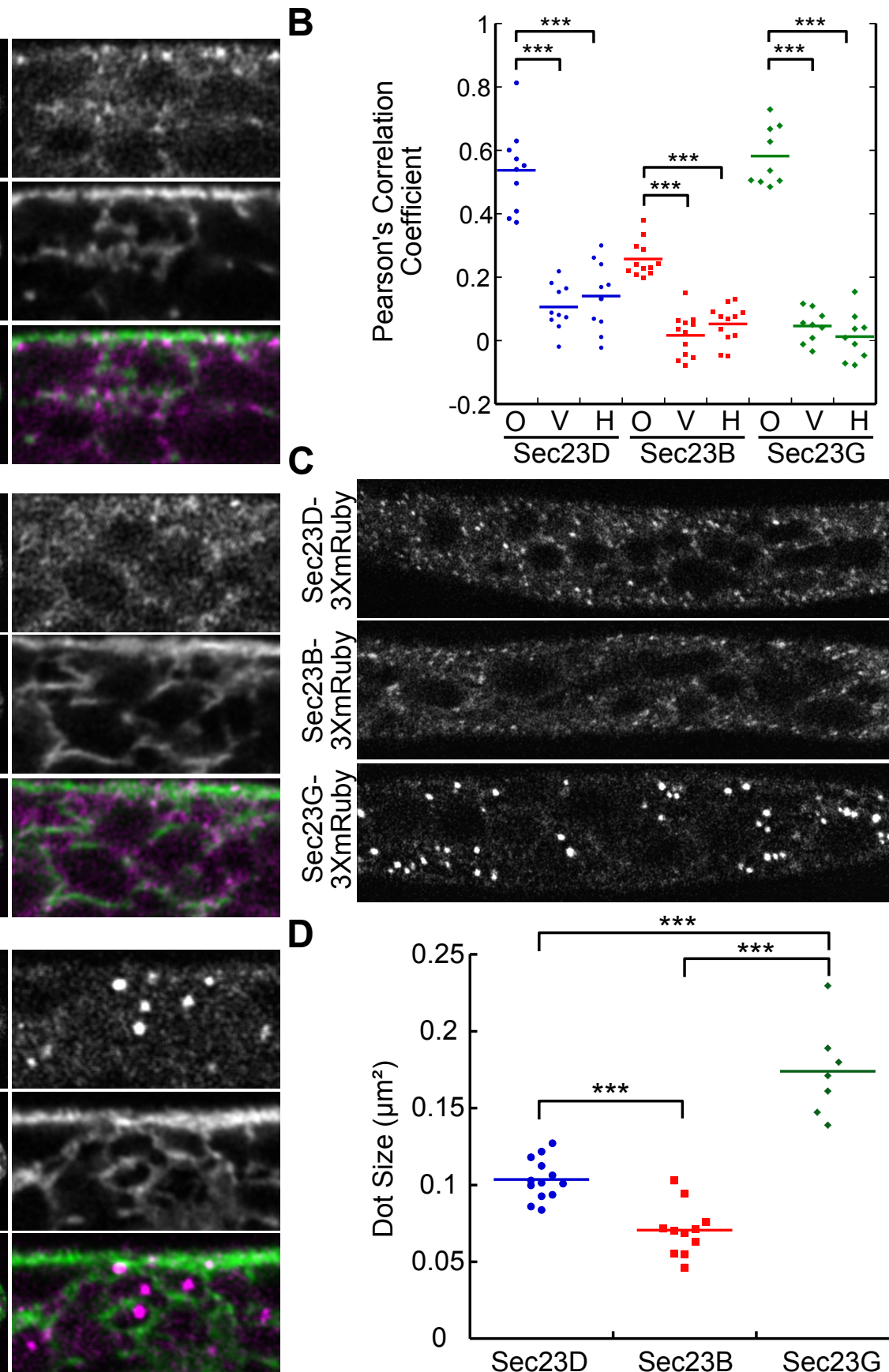

c

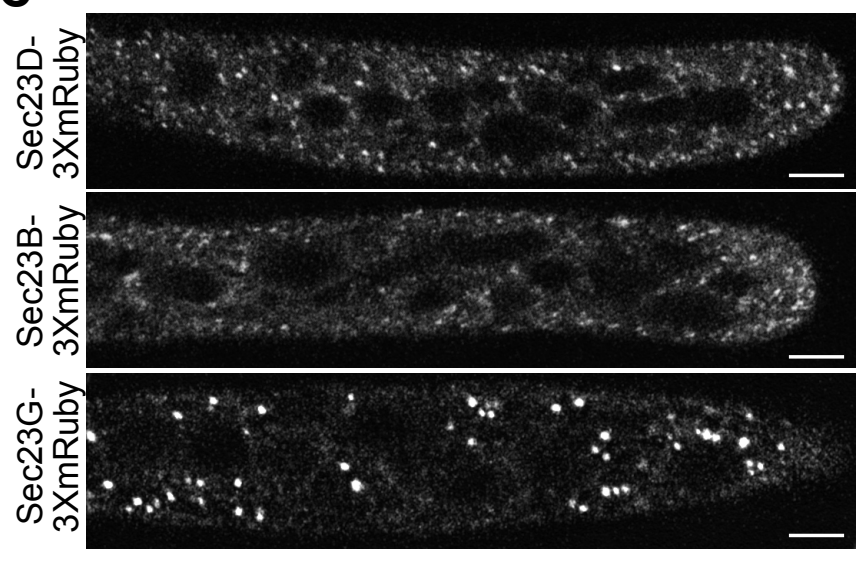

D

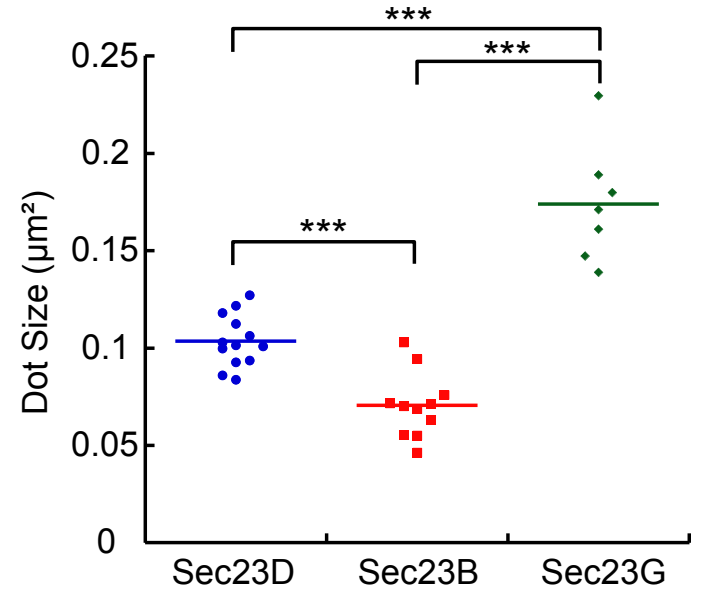

Figure 6. Sec23 isoforms localize to ER proximal structures with distinct sizes. (A) Representative confocal Z-sections of lines where the indicated Sec23 gene was endogenously tagged with 3XmRuby in a line that expresses GFP-KDEL labeling the ER lumen (ER-GFP). Scale bars, $5 \mu \mathrm{m}$. Boxed region in the mRuby channel is shown to the right of each image. (B) Pearson's correlation coefficient measured between the Sec23 labeled lines and ER-GFP. O, original ER image; V, vertically flipped ER image; $H$, horizontally flipped ER image. Asterisks denote $p<0.001$ as determined by a one-way ANOVA with a Tukey post hoc test $(\alpha=0.05)(C)$ Representative confocal Z-sections of Sec23 genes endogenously tagged with 3XmRuby acquired with the optimal resolution of the confocal imaging system. Scale bars, $5 \mu \mathrm{m}$. (D) Quantification of average dot size in the Sec23 images as determined by the diAna tool for Fiji (Gilles et al., 2017). Asterisks denote $p<0.001$ as determined by a one-way ANOVA with a Tukey post hoc test $(\alpha=0.05)$. 


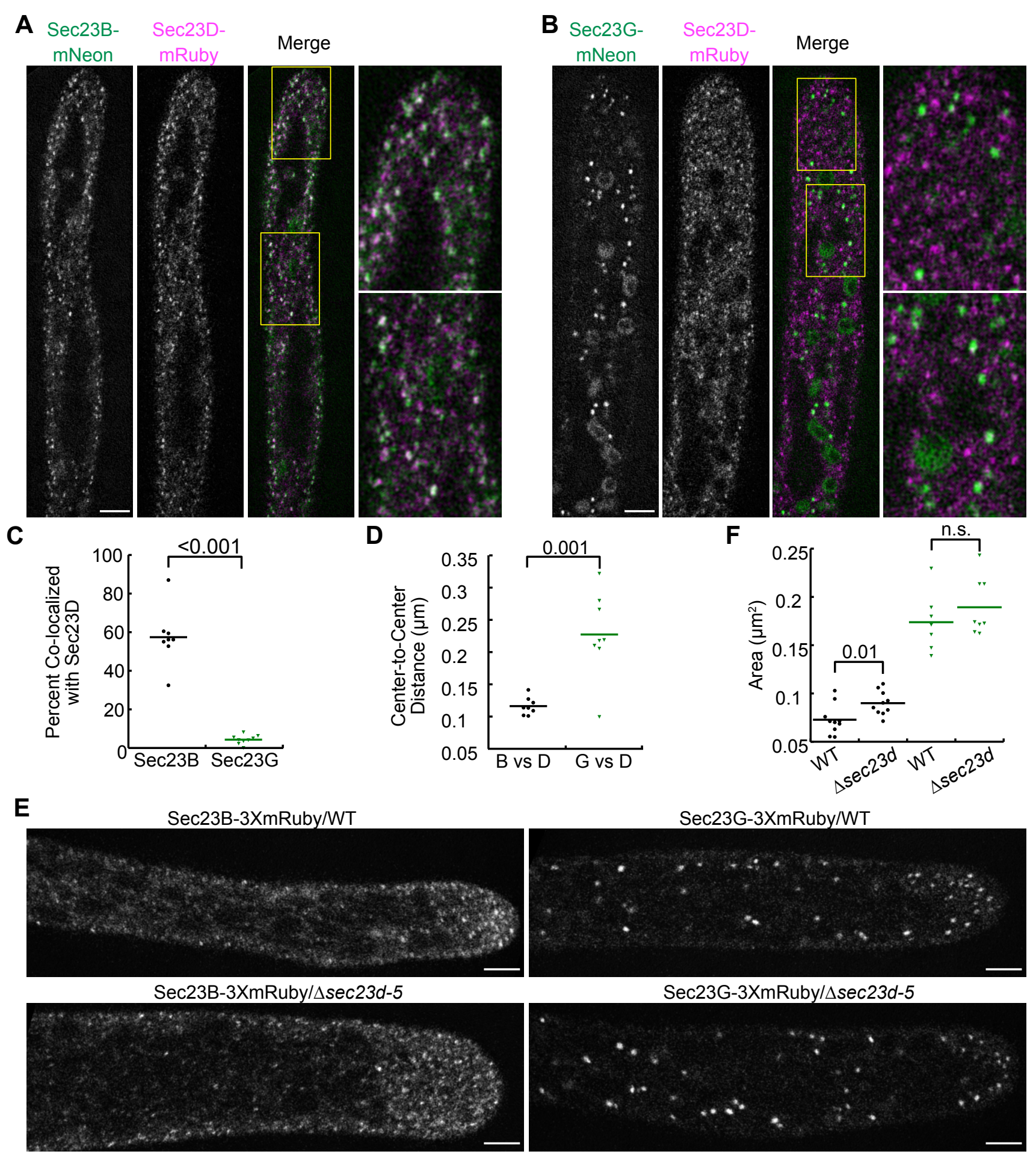

Figure 7. Sec23B and Sec23G form ER exit sites independent of Sec23D. Representative confocal Zsections of Sec23D-mRuby lines where Sec23B (A) or Sec23G (B) were endogenously tagged with mNeon. Scale bar, $5 \mu \mathrm{m}$. Yellow boxed regions are enlarged to the right of the merged image where Sec23D-mRuby is false-colored magenta and in (A) Sec23B-mNeon is false-colored green and in (B) Sec23G-mNeon is false-colored green. (C) Percent of Sec23B-mNeon or Sec23G-mNeon dots that co-localized with Sec23DmRuby and (D) center-to-center distance of co-localized particles as determined using the diAna tool for Fiji (Gilles et al., 2017). (E) Representative confocal Z-sections of wild type and $\Delta s e c 23 d-5$ cells with either Sec23B or Sec23G endogenously tagged with 3XmRuby. Scale bar, $5 \mu \mathrm{m}$. (F) Quantification of average dot size as determined by the diAna tool for Fiji (Gilles et al., 2017). Sec23B and Sec23G dot size in wild type is the same data presented in Figure 6D. Student t-test was performed for data in ( $C, D$, and $F)$. The $t-$ probability is indicated for each data pair in each graph. n.s. denotes no significant difference. 


\section{Parsed Citations}

Aridor, M. (2018). COPII gets in shape: Lessons derived from morphological aspects of early secretion. Traffic 19: 823-839.

Google Scholar: Author Only Title Only Author and Title

Aridor, M., Fish, K.N., Bannykh, S., Weissman, J., Roberts, T.H., Lippincott-Schwartz, J., and Balch, W.E. (2001). The Sar1 Gtpase

Coordinates Biosynthetic Cargo Selection with Endoplasmic Reticulum Export Site Assembly. Journal of Cell Biology 152: 213-230.

Google Scholar: Author Only Title Only Author and Title

Aridor, M., Weissman, J., Bannykh, S., Nuoffer, C., and Balch, W.E. (1998). Cargo Selection by the COPII Budding Machinery during

Export from the ER. Journal of Cell Biology 141: 61-70.

Google Scholar: Author Only Title Only Author and Title

Augustine, R.C., Pattavina, K.A, Tüzel, E., Vidali, L., and Bezanilla, M. (2011). Actin interacting protein1 and actin depolymerizing factor drive rapid actin dynamics in Physcomitrella patens. Plant Cell 23: 3696-3710.

Google Scholar: Author Only Title Only Author and Title

Barlow, L.D. and Dacks, J.B. (2018). Seeing the endomembrane system for the trees: Evolutionary analysis highlights the importance of plants as models for eukaryotic membrane-trafficking. Seminars in Cell \& Developmental Biology 80: 142-152.

Google Scholar: Author Only Title Only Author and Title

Barlowe, C. (2003). Signals for COPII-dependent export from the ER: what's the ticket out? Trends in Cell Biology 13: 295-300.

Google Scholar: Author Only Title Only Author and Title

Barlowe, C. and Helenius, A (2016). Cargo Capture and Bulk Flow in the Early Secretory Pathway. Annu. Rev. Cell Dev. Biol. 32: 197222.

Google Scholar: Author Only Title Only Author and Title

Barlowe, C. and Schekman, R. (1993). SEC12 encodes a guanine-nucleotide-exchange factor essential for transport vesicle budding from the ER. Nature 365: 347-349.

Google Scholar: Author Only Title Only Author and Title

Bascom, C.S., Wu, S.-Z, Nelson, K., Oakey, J., and Bezanilla, M. (2016). Long-Term Growth of Moss in Microfluidic Devices Enables Subcellular Studies in Development. Plant Physiology 172: 28-37.

Google Scholar: Author Only Title Only Author and Title

Belden, W.J. (2001). Role of Erv29p in Collecting Soluble Secretory Proteins into ER-Derived Transport Vesicles. Science 294: 15281531.

Google Scholar: Author Only Title Only Author and Title

Bezanilla, M., Perroud, P.-F., Pan, A, Klueh, P., and Quatrano, R.S. (2005). An RNAi system in Physcomitrella patens with an internal marker for silencing allows for rapid identification of loss of function phenotypes. Plant Biol (Stuttg) 7: 251-257.

Google Scholar: Author Only Title Only Author and Title

Bi, X., Corpina, R.A, and Goldberg, J. (2002). Structure of the Sec23/24-Sar1 pre-budding complex of the COPIl vesicle coat. Nature 419: 271-277.

Google Scholar: Author Only Title Only Author and Title

Brandizzi, F. (2018). Transport from the endoplasmic reticulum to the Golgi in plants: Where are we now? Seminars in Cell \& Developmental Biology 80: 94-105.

Google Scholar: Author Only Title Only Author and Title

Brandizzi, F. and Barlowe, C. (2013). Organization of the ER-Golgi interface for membrane traffic control. Nat Rev Mol Cell Biol 14: 382-392.

Google Scholar: Author Only Title Only Author and Title

Cheng, F-y., Zamski, E., Guo W-w., Mason Pharr, D., and Williamson, J.D. (2009). Salicylic acid stimulates secretion of the normally symplastic enzyme mannitol dehydrogenase: a possible defense against mannitol-secreting fungal pathogens. Planta 230: $1093-1103$.

Google Scholar: Author Only Title Only Author and Title

Cheng, X., and Bezanilla M. (2021). SABRE populates ER domains essential for cell plate maturation and cell expansion influencing cell and tissue patterning. eLife 10: e65166.

Google Scholar: Author Only Title Only Author and Title

Cho, Y. and Kanehara, K. (2017). Endoplasmic Reticulum Stress Response in Arabidopsis Roots. Front. Plant Sci. 8.

Google Scholar: Author Only Title Only Author and Title

Chung, K.P., Zeng, Y., and Jiang, L. (2016). COPII Paralogs in Plants: Functional Redundancy or Diversity? Trends in Plant Science 21: 758-769.

Google Scholar: Author Only Title Only Author and Title

De Craene, J.-O., Courte, F., Rinaldi, B., Fitterer, C., Herranz, M.C., Schmitt-Keichinger, C., Ritzenthaler, C., and Friant, S. (2014). Study of the Plant COPII Vesicle Coat Subunits by Functional Complementation of Yeast Saccharomyces cerevisiae Mutants. PLoS ONE 9: 
e90072.

Google Scholar: Author Only Title Only Author and Title

Ding, Y., Wang, J., Wang, J., Stierhof, Y-D., Robinson, D.G., and Jiang L. (2012). Unconventional protein secretion. Trends in Plant Science 17: 606-615.

Google Scholar: Author Only Title Only Author and Title

Faso, C., Chen, Y.-N., Tamura, K., Held, M., Zemelis, S., Marti, L., Saravanan, R., Hummel, E., Kung, L., Miller, E., Hawes, C., and Brandizzi, F. (2009). A Missense Mutation in the Arabidopsis COPII Coat Protein Sec24AInduces the Formation of Clusters of the Endoplasmic Reticulum and Golgi Apparatus. The Plant Cell 21: 3655-3671.

Google Scholar: Author Only Title Only Author and Title

Fath, S., Mancias, J.D., Bi, X., and Goldberg, J. (2007). Structure and Organization of Coat Proteins in the COPII Cage. Cell 129: 13251336.

Google Scholar: Author Only Title Only Author and Title

Furt, F., Lemoi, K., Tüzel, E., and Vidali, L. (2012). Quantitative analysis of organelle distribution and dynamics in Physcomitrella patens protonemal cells. BMC Plant Biol. 12: 70.

Google Scholar: Author Only Title Only Author and Title

Galotto, G., Bibeau, J.P., and Vidali, L. (2019). Automated Image Acquisition and Morphological Analysis of Cell Growth Mutants in Physcomitrella patens. In Plant Cell Morphogenesis, F. Cvrčková and V. Žárský, eds, Methods in Molecular Biology. (Springer New York: New York, NY), pp. 307-322.

Google Scholar: Author Only Title Only Author and Title

Gilles, J.-F., Dos Santos, M., Boudier, T., Bolte, S., and Heck, N. (2017). DiAna, an ImageJ tool for object-based 3D co-localization and distance analysis. Methods 115: 55-64.

Google Scholar: Author Only Title Only Author and Title

Giraudo, C.G. and Maccioni, H.J.F. (2003). Endoplasmic Reticulum Export of Glycosyltransferases Depends on Interaction of a Cytoplasmic Dibasic Motif with Sar1. MBoC 14: 3753-3766.

Google Scholar: Author Only Title Only Author and Title

van Gisbergen, P.AC., Wu, S.-Z, Chang, M., Pattavina, K.A, Bartlett, M.E., and Bezanilla, M. (2018). An ancient Sec10-formin fusion provides insights into actin-mediated regulation of exocytosis. J. Cell Biol. 217: 945-957.

Google Scholar: Author Only Title Only Author and Title

Haeussler, M., Schönig, K., Eckert, H., Eschstruth, A, Mianné, J., Renaud, J.-B., Schneider-Maunoury, S., Shkumatava, A, Teboul, L., Kent, J., Joly, J.-S., and Concordet, J.-P. (2016). Evaluation of off-target and on-target scoring algorithms and integration into the guide RNA selection tool CRISPOR. Genome Biol. 17: 148.

Google Scholar: Author Only Title Only Author and Title

Hanna, M.G., Peotter, J.L., Frankel, E.B., and Audhya, A (2018). Membrane Transport at an Organelle Interface in the Early Secretory Pathway: Take Your Coat Off and Stay a While: Evolution of the metazoan early secretory pathway. BioEssays 40: 1800004.

Google Scholar: Author Only Title Only Author and Title

Hanton, S.L., Matheson, L.A, Chatre, L., and Brandizzi, F. (2009). Dynamic organization of COPII coat proteins at endoplasmic reticulum export sites in plant cells. The Plant Journal 57: 963-974.

Google Scholar: Author Only Title Only Author and Title

Heinze, L., Freimuth, N., Rößling, A-K., Hahnke, R., Riebschläger, S., Fröhlich, A, Sampathkumar, A, McFarlane, H.E., and Sauer, M. (2020). EPSIN1 and MTV1 define functionally overlapping but molecularly distinct trans-Golgi network subdomains in Arabidopsis.

PNAS 117: 25880-25889.

Google Scholar: Author Only Title Only Author and Title

Hino, T., Tanaka, Y., Kawamukai, M., Nishimura, K., Mano, S., and Nakagawa, T. (2011). Two Sec13p Homologs, AtSec13A and AtSec13B, Redundantly Contribute to the Formation of COPII Transport Vesicles in Arabidopsis thaliana. Bioscience, Biotechnology, and Biochemistry 75: 1848-1852.

Google Scholar: Author Only Title Only Author and Title

Hiwatashi, Y., Sato, Y., and Doonan, J.H. (2014). Kinesins Have a Dual Function in Organizing Microtubules during Both Tip Growth and Cytokinesis in Physcomitrella patens. The Plant Cell 26: 1256-1266.

Google Scholar: Author Only Title Only Author and Title

Jensen, D. and Schekman, R. (2011). COPII-mediated vesicle formation at a glance. Journal of Cell Science $124: 1-4$.

Google Scholar: Author Only Title Only Author and Title

Khoriaty, R. et al. (2018). Functions of the COPII gene paralogs SEC23Aand SEC23B are interchangeable in vivo. Proc Natl Acad Sci USA 115: E7748-E7757.

Google Scholar: Author Only Title Only Author and Title

Lee, M.H., Lee, S.H., Kim, H., Jin, J.B., Kim, D.H., and Hwang, I. AWD40 Repeat Protein, Arabidopsis Sec13 Homolog 1, May Play a Role in Vacuolar Trafficking by Controlling the Membrane Association of AtDRP2A: 10. 
Mallett, D.R., Chang, M., Cheng, X., and Bezanilla, M. (2019). Efficient and modular CRISPR-Cas9 vector system for Physcomitrella patens. Plant Direct 3: e00168-15.

Google Scholar: Author Only Title Only Author and Title

Mancias, J.D. and Goldberg, J. (2007). The Transport Signal on Sec22 for Packaging into COPII-Coated Vesicles Is a Conformational Epitope. Molecular Cell 26: 403-414.

Google Scholar: Author Only Title Only Author and Title

Maruyama, D., Sugiyama, T., Endo, T., and Nishikawa, S. (2014). Multiple BiP Genes of Arabidopsis thaliana are Required for Male

Gametogenesis and Pollen Competitiveness. Plant and Cell Physiology 55: 801-810.

Google Scholar: Author Only Title Only Author and Title

Mossessova, E., Bickford, L.C., and Goldberg, J. (2003). SNARE selectivity of the COPII coat. Cell 114: 483-495.

Google Scholar: Author Only Title Only Author and Title

Nakano, A and Muramatsu, M. (1989). ANovel GTP-binding Protein, Sarlp, Is Involved in Transport from the Endoplasmic Reticulum to the Golgi Apparatus. The Journal of Cell Biology 109: 2677-2691.

Google Scholar: Author Only Title Only Author and Title

Nakano, R.T., Matsushima, R., Ueda, H., Tamura, K., Shimada, T., Li, L., Hayashi, Y., Kondo, M., Nishimura, M., and Hara-Nishimura, I. (2009). GNOM-LIKE1/ERMO1 and SEC24a/ERMO2 Are Required for Maintenance of Endoplasmic Reticulum Morphology in Arabidopsis thaliana. The Plant Cell 21: 3672-3685.

Google Scholar: Author Only Title Only Author and Title

Nebenfuhr, A (2002). Brefeldin A: Deciphering an Enigmatic Inhibitor of Secretion. Plant physiology 130: 1102-1108.

Google Scholar: Author Only Title Only Author and Title

Nelson, B.K., Cai, X., and Nebenführ, A (2007). Amulticolored set of in vivo organelle markers for co-localization studies in

Arabidopsis and other plants. Plant J. 51: 1126-1136.

Google Scholar: Author Only Title Only Author and Title

Niu, T.-K., Pfeifer, AC., Lippincott-Schwartz, J., and Jackson, C.L. (2005). Dynamics of GBF1, a Brefeldin A-Sensitive Arf1 Exchange Factor at the Golgi $\square$ V. Molecular Biology of the Cell 16: 1213-1222.

Google Scholar: Author Only Title Only Author and Title

Noh, S.-J., Kwon, C.S., Oh, D.-H., Moon, J.S., and Chung, W.-I. (2003). Expression of an evolutionarily distinct novel BiP gene during the unfolded protein response in Arabidopsis thaliana. Gene 311: 81-91.

Google Scholar: Author Only Title Only Author and Title

Novick, P., Field, C., and Schekman, R. (1980). Identification of 23 complementation groups required for post-translational events in the yeast secretory pathway. Cell 21: 205-215.

Google Scholar: Author Only Title Only Author and Title

Nufer, O. (2003). ER export of ERGIC-53 is controlled by cooperation of targeting determinants in all three of its domains. Journal of Cell Science 116: 4429-4440.

Google Scholar: Author Only Title Only Author and Title

Ortiz-Ramírez, C., Michard, E., Simon, AA, Damineli, D.S.C., Hernandez-Coronado, M., Becker, J.D., and Feijó, J.A (2017). GLUTAMATE RECEPTOR-LIKE channels are essential for chemotaxis and reproduction in mosses. Nature 549: 91-95.

Google Scholar: Author Only Title Only Author and Title

Qu, X., Zhang, R., Zhang, M., Diao, M., Xue, Y., and Huang, S. (2017). Organizational innovation of apical actin filaments drives rapid pollen tube growth and turning. Mol Plant 10: 930-947.

Google Scholar: Author Only Title Only Author and Title

Rensing, S.A, Goffinet, B., Meyberg, R., Wu, S.-Z, and Bezanilla, M. (2020). The Moss Physcomitrium( Physcomitrella ) patens : A Model Organism for Non-Seed Plants. Plant Cell 32: 1361-1376.

Google Scholar: Author Only Title Only Author and Title

Robinson, D.G., Brandizzi, F., Hawes, C., and Nakano, A (2015). Vesicles versus Tubes: Is Endoplasmic Reticulum-Golgi Transport in Plants Fundamentally Different from Other Eukaryotes? Plant Physiology 168: 393-406.

Google Scholar: Author Only Title Only Author and Title

Salama, N.R., Yeung, T., and Schekman, R.W. The Secl3p complex and reconstitution of vesicle budding from the ER with purified cytosolic proteins. The EMBO Journal 12: 4073-4082.

Google Scholar: Author Only Title Only Author and Title

Scheuring, D., Schöller, M., Kleine-Vehn, J., and Löfke, C. (2015). Vacuolar staining methods in plant cells. Methods Mol Biol 1242: 8392.

Google Scholar: Author Only Title Only Author and Title

Schlacht, A and Dacks, J.B. (2015). Unexpected Ancient Paralogs and an Evolutionary Model for the COPII Coat Complex. Genome Biology and Evolution 7: 1098-1109. 
Srivastava, R., Deng, Y., Shah, S., Rao, AG., and Howell, S.H. (2013). BINDING PROTEIN Is a Master Regulator of the Endoplasmic Reticulum Stress Sensor/Transducer bZP28 in Arabidopsis. Plant Cell 25: 1416-1429.

Google Scholar: Author Only Title Only Author and Title

Stagg, S.M., Gürkan, C., Fowler, D.M., LaPointe, P., Foss, T.R., Potter, C.S., Carragher, B., and Balch, W.E. (2006). Structure of the Sec13/31 COPII coat cage. Nature 439: 234-238.

Google Scholar: Author Only Title Only Author and Title

Stagg, S.M., LaPointe, P., Razvi, A, Gürkan, C., Potter, C.S., Carragher, B., and Balch, W.E. (2008). Structural Basis for Cargo Regulation of COPII Coat Assembly. Cell 134: 474-484.

Google Scholar: Author Only Title Only Author and Title

Takagi, J., Renna, L., Takahashi, H., Koumoto, Y., Tamura, K., Stefano, G., Fukao, Y., Kondo, M., Nishimura, M., Shimada, T., Brandizzi, F., and Hara-Nishimura, I. (2013). MAIGO5 Functions in Protein Export from Golgi-Associated Endoplasmic Reticulum Exit Sites in

Arabidopsis. The Plant Cell 25: 4658-4675.

Google Scholar: Author Only Title Only Author and Title

Tanaka, Y., Nishimura, K., Kawamukai, M., Oshima, A, and Nakagawa, T. (2013). Redundant function of two Arabidopsis COPII components, AtSec24B and AtSec24C, is essential for male and female gametogenesis. Planta 238: 561-575.

Google Scholar: Author Only Title Only Author and Title

Vidali, L., Augustine, R.C., Kleinman, K.P., and Bezanilla, M. (2007). Profilin is essential for tip growth in the moss Physcomitrella patens. Plant Cell 19: 3705-3722.

Google Scholar: Author Only Title Only Author and Title

Vidali, L., van Gisbergen, P.AC., Guérin, C., Franco, P., Li, M., Burkart, G.M., Augustine, R.C., Blanchoin, L., and Bezanilla, M. (2009). Rapid formin-mediated actin-filament elongation is essential for polarized plant cell growth. Proc. Natl. Acad. Sci. U.S.A 106: 1334113346.

Google Scholar: Author Only Title Only Author and Title

Viotti, C. et al. (2013). The Endoplasmic Reticulum Is the Main Membrane Source for Biogenesis of the Lytic Vacuole in Arabidopsis. Plant Cell 25: 3434-3449.

Google Scholar: Author Only Title Only Author and Title

Wang, X., Chung, K. P., Lin, W., and Jiang L. (2017). Protein secretion in plants: conventional and unconventional pathways and new techniques. Journal of Experimental Botany 69: 21-37.

Google Scholar: Author Only Title Only Author and Title

Whittle, J.R.R. and Schwartz, T.U. (2010). Structure of the Sec13-Sec16 edge element, a template for assembly of the COPII vesicle coat. The Journal of Cell Biology 190: 347-361.

Google Scholar: Author Only Title Only Author and Title

Wu, S.-Z and Bezanilla, M. (2014). Myosin VII associates with microtubule ends and together with actin plays a role in guiding plant cell division. eLife Sciences 3: e03498.

Google Scholar: Author Only Title Only Author and Title

Wu, S.-Z, Yamada, M., Mallett, D.R., and Bezanilla, M. (2018). Cytoskeletal discoveries in the plant lineage using the moss Physcomitrella patens. Biophys Rev 10: 1683-1693.

Google Scholar: Author Only Title Only Author and Title

Zeng, Y., Chung, K.P., Li, B., Lai, C.M., Lam, S.K., Wang, X., Cui, Y., Gao, C., Luo, M., Wong, K.-B., Schekman, R., and Jiang, L. (2015). Unique COPII component AtSar1a/AtSec23a pair is required for the distinct function of protein ER export in Arabidopsis thaliana. Proc Natl Acad Sci USA 112: 14360-14365.

Google Scholar: Author Only Title Only Author and Title

Zeng, Y., Li, B., Ji, C., Feng, L., Niu, F., Deng, C., Chen, S., Lin, Y., Cheung, K.C.P., Shen, J., Wong, K.-B., and Jiang, L. (2021). Aunique AtSar1D-AtRabD2a nexus modulates autophagosome biogenesis in Arabidopsis thaliana. Proc Natl Acad Sci U S A118.

Google Scholar: Author Only Title Only Author and Title

Zhang, H., Zhang, L., Gao, B., Fan, H., Jin, J., Botella, M.A, Jiang, L., and Lin, J. (2011). Golgi apparatus-localized synaptotagmin 2 is required for unconventional secretion in Arabidopsis. PLoS ONE 6: e26477.

Google Scholar: Author Only Title Only Author and Title 